\title{
Splicing buffers suboptimal codon usage in human cells
}

Christine Mordstein ${ }^{1,2}$, Rosina Savisaar ${ }^{2,3}$, Robert S Young², Jeanne Bazile1, Lana

Talmane ${ }^{1}$, Juliet Luft ${ }^{1}$, Michael Liss ${ }^{4}$, Martin S Taylor ${ }^{1}$, Laurence D Hurst ${ }^{2}$, Grzegorz Kudla $1^{*}$

${ }^{1}$ MRC Human Genetics Unit, Institute for Genetics and Molecular Medicine, The University of Edinburgh, Scotland, UK

${ }^{2}$ Milner Centre for Evolution, Department of Biology and Biochemistry, University of Bath, Bath, UK

${ }^{3}$ School of Communication and Information Technology, Nile University, Giza, Egypt

${ }^{4}$ Thermo Fisher Scientific, GENEART GmbH, Regensburg, Germany

4,024 words (main text)

\section{Figures}

6 Supplementary Figures

2 Supplementary Tables

*Corresponding author: Grzegorz Kudla (gkudla@gmail.com) 


\section{Abstract}

3 Although multiple studies have addressed the effects of codon usage on gene

4 expression, such studies were typically performed in unspliced model genes. In

5 the human genome, most genes undergo splicing and patterns of codon usage are

6 splicing-dependent: guanine and cytosine (GC) content is highest within single-

7 exon genes and within first exons of multi-exon genes. Intrigued by this

8 observation, we measured the effects of splicing on expression in a panel of

9 synonymous variants of GFP and mKate2 reporter genes that varied in

10 nucleotide composition. We found that splicing promotes the expression of

11 adenine and thymine (AT)-rich variants by increasing their steady-state protein

12 and mRNA levels, in part through promoting cytoplasmic localization of mRNA.

13 Splicing had little or no effect on the expression of GC-rich variants. In the 14 absence of splicing, high GC content at the 5 ' end, but not at the 3 ' end of the

15 coding sequence positively correlated with expression. Among endogenous 16 human protein-coding transcripts, GC content has a more positive effect on 17 various expression measures of unspliced, relative to spliced mRNAs. We 18 propose that splicing promotes the expression of AT-rich genes, leading to 19 selective pressure for the retention of introns in the human genome. 


\section{Introduction}

Mammalian genomes are characterised by large regional variation in base composition (Bernardi, 1993). Regions with a high density of G and C nucleotides (GC-rich regions) are in an open, transcriptionally active state, are gene-dense, and replicate early. In contrast, AT-rich regions are enriched with heterochromatin, contain large gene deserts and replicate late (Arhondakis et al., 2011; Lander et al., 2001; Vinogradov, 2003). The mechanisms that give rise to this compositional heterogeneity have been under debate for years and many researchers believe that the pattern originates from the process of GC-biased gene conversion (Duret and Galtier, 2009), though other neutral and selective mechanisms have been proposed as well (Eyre-Walker, 1991; Galtier et al., 2018; Plotkin and Kudla, 2011; Sharp and Li, 1987b).

The sequence composition of mammalian genes correlates with the GC-content of their genomic location. Thus, introns and exons of genes located in GC-rich parts of the genome are themselves GC-rich. This can potentially influence gene expression in multiple ways: nucleotide composition affects the physical properties of DNA, the thermodynamic stability of RNA folding, the propensity of RNA to interact with other RNAs and proteins, the codon adaptation of mRNA to tRNA pools, and the propensity for RNA modifications, such as m6A (Dominissini et al., 2012) and ac4C (Arango et al., 2018). Strikingly, studies of the effects of nucleotide composition on gene expression in human cells have led to opposing conclusions. On the one hand, heterologous expression experiments typically report large positive effects of GC content on protein production in a wide variety of transgenes, including fluorescent reporter genes, human cDNAs, and viral genes (Bauer et al., 2010; Kosovac et al., 2011; Kotsopoulou et al., 2000; Kudla et al., 2006; Zolotukhin et al., 1996). As a result, increasing the GC content of transgenes has become a common strategy in coding sequence optimization

50 for heterologous expression in human cells (Fath et al., 2011). On the other hand, 51 genome-wide analyses of endogenous genes typically show little or no 52 correlation of GC content with expression (Duan et al., 2013; Lercher et al., 2003;

53 Rudolph et al., 2016; Semon et al., 2005). 
55 We hypothesized that the conflicting results in heterologous and endogenous gene expression studies can be partially explained by RNA splicing. Most transgenes used in heterologous expression systems have no introns, whereas $97 \%$ of genes in the human genome contain one or more introns. Splicing is known to influence gene expression at multiple stages, including nuclear RNP assembly, RNA export, and translation. If splicing selectively increased the expression of AT-rich genes, it could account for the lack of correlation of GC content and gene expression in previous genome-wide studies. We therefore compared spliced and unspliced genes with respect to their (1) genomic codon usage, (2) expression levels of reporter genes in transient and stable transfection experiments and (3) global expression patterns in human transcriptome studies. We show that splicing increases the expression of AT-rich genes, but not GC-rich genes, in part through effects on cytoplasmic RNA enrichment.

\section{Results}

\section{Codon usage of human protein-coding genes depends on RNA splicing}

We first analysed the relationship between the nucleotide composition of human genes and splicing. GC4 content (GC content at 4-fold degenerate sites) correlates negatively with the number of exons in humans (Figure 1A; Spearman's $\rho=$ -0.27; p < 2.2×10-16; see also (Carels and Bernardi, 2000; Ressayre et al., 2015; Savisaar and Hurst, 2016)). In addition, GC4 content is highest in 5'-proximal exons (Figure 1B; Spearman's $\rho=-0.18 ; \mathrm{p}<2.2 \times 10^{-16}$ ), and first exons have a higher GC4 content than second exons ( $\mathrm{p}<2.2 \times 10^{-16}$, one-tailed Wilcoxon test). Although these patterns could result from proximity to $\mathrm{CpG}$-rich transcription start sites (TSSs)(Zhang et al., 2004), we found that first exons have significantly

81 higher GC4 content than second exons even when controlling for the distance from the TSS (Figure 1C). This suggests that splicing contributes to the observed enrichment of $\mathrm{G}$ and $\mathrm{C}$ nucleotides in the 5'-proximal exons in human.

85 To understand the causal links between splicing and nucleotide composition, we studied the compositional patterns of retrogenes. Retrotransposition provides a 
natural evolutionary experiment of what happens when a previously spliced gene suddenly loses its introns. We first analysed a set of 49 parent-retrogene pairs for which both the parent and the retrocopy ORFs have been retained in human and mouse. Strikingly, we found that the retrocopies had a significantly higher GC4 content than their parents (median GC4 $4_{\text {retrocopy }}-$ GC4 $_{\text {parent }}=11.5 \%$; $=2.1 \times 10^{-4}$ from one-tailed Wilcoxon test; Figure 1D). It thus appears that after retrotransposition, newly integrated intronless genes come under selective pressure for increased GC content. In a comparison of 31 parent-retrogene pairs retained between human and macaque, the median GC4 difference is not significant $(0.09 \%$; $p=0.13$, Wilcoxon test), but this may be explained by duplication events in macaques being more recent $(\mathrm{dS} \sim 0.06)$ than in mouse $(\mathrm{dS}$ $\sim 0.5$ ) and therefore less evolutionary time has passed to allow changes in GC composition to have occurred. As a control, we analysed the GC4 content of retrocopies classed as pseudogenes (Figure S1A) and found it to be significantly lower compared to their parental genes (-2.963\%; $\mathrm{p}<2.2 \times 10^{-16}$, Wilcoxon test). Furthermore, the genomic neighbourhood of functional retrocopies and pseudogenes had significantly lower GC content than the neighbourhood of their respective parental genes (Figure S1B). These observations suggest that increased GC content is not intrinsically connected with retrotransposition, but is required for maintaining long-term functionality of retrogenes. Taken together, these results support a splicing-dependent mechanism shaping conserved patterns of nucleotide composition across functional protein-coding genes.

\section{GC-content is a strong predictor of expression of unspliced reporter genes}

The above analyses show a connection between splicing and genomic GC content of endogenous human genes. To test whether splicing differentially affects the expression of genes depending on their GC content, we designed 22 synonymous variants of GFP that span a broad range of GC3 content (GC content at the third positions of codons) (Mittal et al., 2018) (Figure S2). The collection encompasses most of the variation in GC3 content found among human genes. All variants were independently designed by randomly drawing each codon from an appropriate probability distribution, to ensure uniform GC content and statistical 
120 independence between sequences. We cloned these variants into two

121 mammalian expression vectors: an intronless vector with a CMV promoter

122 (рCM3) and a version of the same vector with a synthetic intron located in the 5'

123 UTR (pCM4). The GC content profiles of the 5' UTRs were similar in both vectors

124 (Figure S2E,F). The vectors also encoded a far-red fluorescent protein, mKate2,

125 which we used to normalize GFP protein abundance (normalization reduced

126 measurement noise, but similar results were obtained with and without

127 normalization). Transient transfections of HeLa cells with three independent

128 preparations of each plasmid showed reproducible expression with a large

129 dynamic range: synonymous variants differed in GFP protein production 46-fold.

130 Consistent with previous studies, GFP fluorescence was strongly correlated with

131 GC3 content, both in spliced and unspliced genes (Figure 2A,B). Interestingly,

132 introduction of an intron into the 5' UTR increased the expression of most, but

133 not all variants. Typically, GC-poor variants experienced a large increase of

134 expression in the presence of an intron, whereas GC-rich variants were 135 unaffected or experienced a moderate increase (Figure 2C).

137 We obtained similar results in stably transfected HeLa and HEK293 cells (Figure 138 S3) and when expressing an independently designed collection of 25 139 synonymous variants of mKate2 in HeLa cells (Figure 2D-F, S2D). A Fisher's 140 exact test revealed that the percentage of variants whose expression was 141 increased by splicing significantly depended on GC3 content ( $p=0.02, N=47$, GFP 142 and mKate variants combined). These experiments show that many AT-rich 143 genetic variants are expressed inefficiently in human cells, but low expression 144 can be partially rescued by splicing. Notably, the average GC content of the 145 human genome is $41 \%$ (Li, 2011). In our experiments, genes with GC content 146 below 41\% are expressed extremely inefficiently, unless they contain an intron 147 (Figure 2). This may provide a strong selective pressure for the retention of 148 introns in many human genes.

150 To establish which stages of expression are responsible for this phenomenon, we 151 first measured mRNA abundance of GFP variants in transiently transfected HeLa 152 cells by quantitative RT-PCR (qRT-PCR). High GC content may introduce 
153 unwanted bias in RT-PCR, so to allow fair comparison of all variants irrespective

154 of their GC content, qRT-PCR primers were placed in the untranslated regions,

155 whose sequence did not vary. Similar to protein levels, mRNA abundance varied 156 widely between synonymous variants of GFP. GC-poor variants experienced a

157 large increase of expression in the presence of an intron, whereas GC-rich 158 variants were less affected (Figure 2G-I). The range of variation in mRNA 159 abundance was much smaller in constructs with an intron than without intron 160 (Figure 2I), indicating that splicing buffers the effects of GC content on 161 expression.

163 We then asked if changes in mRNA abundance arose at transcriptional or post164 transcriptional levels. As a proxy for transcriptional efficiency, we measured the 165 abundance of intronic RNA for GFP variants expressed from the intron166 containing plasmid. GC content did not correlate with intronic RNA abundance 167 (Figure 2J), suggesting that the rate of transcription does not depend on GC content of the coding sequence found downstream of the intron. Conversely, high GC content was associated with stabilization in unspliced constructs (Figure 2K).

170 Taken together, these experiments show that splicing preferentially increases

171 the expression of GC-poor synonymous variants at a post-transcriptional level.

\section{High GC content at the $5^{\prime}$ end correlates with efficient expression}

174 To further explore the sequence determinants of expression, we assembled a 175 pool of 217 synonymous variants of GFP that included the 22 variants studied 176 above, 137 variants from our earlier study (Kudla et al., 2009), and 58 additional 177 variants. We cloned the collection into plasmids with and without a 5' UTR 178 intron. We then established pools of HeLa Flp-In T-REx cells that stably express 179 these constructs from a single genomic locus under a doxycycline-inducible 180 promoter and measured the protein levels of all variants by Flow-Seq (Kosuri et 181 al., 2013). We also performed Flow-Seq in HEK293 cells using the intronless constructs only. In Flow-Seq, a pool of cells is sorted by FACS into bins of 183 increasing fluorescence and the distribution of variants in each bin is probed by 184 amplicon sequencing to quantify protein abundance (Figure 3A). All variants 185 could be quantified with good technical and biological reproducibility and high 
186

187

188

189

190

191

192

193

194

195

196

197

198

199

200

201

202

203

204

205

206

correlation was found between Flow-Seq and spectrofluorometric measurement of individual constructs (Figure S4). Most variants showed the expected unimodal distribution across fluorescence bins, but some variants showed bimodal distributions, possibly indicative of gene silencing in a fraction of cells.

All Flow-Seq experiments showed substantial variation of expression between synonymous variants of GFP (Figure 3B). GFP protein levels in HeLa cells (with intron), HeLa cells (without intron), and HEK293 cells (without intron) were all correlated with each other, but the moderate degree of correlation $(r=0.51$ HEK293 (without intron) vs HeLa (without intron); r=0.36 Hela (with intron) vs HeLa (without intron)) suggests that the effects of codon usage on expression are modulated by splicing and by cell line identity - in agreement with prior observations of tissue-specific codon usage (Burow et al., 2018; Gingold et al., 2014; Plotkin et al., 2004; Rudolph et al., 2016). Flow-Seq of unspliced variants in HeLa and HEK cells confirms the positive correlation of synonymous site GCcontent with expression (Figure 3C). In contrast to the results reported by us and others in bacteria and yeast (Goodman et al., 2013; Kudla et al., 2009; Shah et al., 2013), but consistently with the positive correlation between GC content and expression, strong mRNA folding near the beginning of the coding sequence correlated with increased expression (Spearman's $\rho=0.27$ in HeLa cells; $\rho=0.4$ in HEK293 cells). Expression was positively correlated with CpG content and codon adaptation index (CAI), and negatively correlated with the estimated density of ARE elements or cryptic splice sites. Because of the strong correlation between GC content, $\mathrm{CpG}$ content, $\mathrm{CAI}$ and mRNA folding energy, a multiple regression analysis could not resolve which of these properties was causally related to expression. Interestingly, for intron-containing variants, there was no correlation, or a weak negative correlation, between expression and GC content, CpG content, CAI, and mRNA folding energy (Figure 3C).

Some of the variants analysed by Flow-Seq featured large regional variation in GC content (Figure S5A) and we asked whether the localization of low-GC and high-GC regions within the coding sequence influences expression. We found that the GC3 content in the first half of the coding sequence (nt 1-360), but not in 
219 the second half (nt 361-720), was positively correlated with expression of

220 intronless GFP variants in the HeLa and HEK293 cells (Figure 3D). The GC3

221 content in the first half of the gene showed no correlation with expression in the

222 intron-containing constructs.

224 To further test whether the GC content at the $5^{\prime}$ end of genes has a particularly 225 important effect on expression, we constructed in-frame fusions between GC226 rich and GC-poor variants of GFP and mKate2 genes and quantified their protein 227 and mRNA abundance in transient transfection experiments. Expression levels 228 showed a striking dependence on the GC content profile. mKate2_GCpoor 229 showed undetectable expression on its own or as a 5'-terminal fusion with GC230 rich GFP, but it was efficiently expressed as a 3'-terminal fusion with GC-rich GFP 231 (Figure 3E). By contrast, mKate2_GCrich was efficiently expressed both as 5'232 terminal and 3'-terminal fusion. Analogous experiments with GC-rich and GC233 poor variants of GFP fused to mKate2_GCrich led to similar conclusions (Figure 234 S5B). The differences in protein levels between the fusion constructs could be 235 explained by differences in mRNA abundance (Figure 3E).

High GC content leads to cytoplasmic enrichment of mRNA and higher

239 Using the pooled HeLa cells used in Flow-Seq, we then analysed the effects of GC 240 content on mRNA localization. We separated the pools into nuclear and 241 cytoplasmic fractions, isolated RNA and performed amplicon sequencing of each 242 fraction to analyse mRNA localization of each GFP variant. Analysis of fractions 243 showed the expected enrichment of the IncRNA MALAT1 in the nucleus, and of 244 tRNA in the cytoplasm, confirming the quality of fractionations (Figure 4A). For 245 each GFP variant, we calculated the relative cytoplasmic concentration of its 246 mRNA (RCC) as the ratio of cytoplasmic read counts to the sum of reads from 247 both fractions (RCC = c_cyto / (c_cyto+c_nuc); Figure 4B). A value of 0 therefore 248 indicates $100 \%$ nuclear retention, whereas a value of 1 indicates $100 \%$ 249 cytoplasmic localisation. In the absence of splicing, RCC scores ranged from 0.09 250 to 0.64 and RCC correlated significantly with GC content $\left(r=0.51, p=3.85 \times 10^{-13}\right.$, 251 Figure 4C). In the presence of a 5' UTR intron, we observed a significant increase 
in RCC score for GFP variants with low GC content, but no increase in RCC for GCrich variants (Figure 4D). GC3 content at the beginning of the coding sequence was significantly correlated with RCC in the absence of splicing $\left(r=0.5, p=2.0 \times 10^{-}\right.$ $\left.{ }^{11}\right)$, but not in the presence of splicing $(\mathrm{R}<0.01, \mathrm{p}=0.48$; Figure S5). Thus, high GC content at the $5^{\prime}$ end of genes increases gene expression in part through facilitating the cytoplasmic localization of mRNA.

To assess whether GC content also affects translational dynamics, we performed polysome profiling on HEK293 GFP pool cells using sucrose gradient fractionation (Figure 5A). qRT-PCR analyses of RNA extracted from all collected fractions showed a broad distribution of GFP across fractions, with enrichment within polysome-associated fractions. In order to determine distribution patterns of individual GFP variants, RNA from several fractions was pooled (as indicated in Figure 5B) and subjected to high-throughput sequencing. The resulting read distribution indicates that GC-rich variants are associated with denser polysomal fractions (ribosome density, Figure 5C, left panel; $\mathrm{R}^{2}=0.55$, $\mathrm{p}<$ $2.2 \times 10^{-16}$ ) and are more likely to be translated (ribosome association, Figure 5C, right panel; $\mathrm{R}^{2}=0.28, \mathrm{p}<9.03 \times 10^{-15}$ ), compared to $\mathrm{GC}$-poor variants. This suggests that enhanced translational dynamics also contribute to more efficient expression of GC-rich genes.

\section{The expression fate of endogenous RNA depends on splicing, nucleotide} composition, and cell type

To test whether splicing- and position-dependent effects of codon usage can also be observed among human genes, we turned to genome-wide measurements of expression at endogenous human loci and related these measurements to codon usage and splicing. Although the correlations between GC content and expression depended on the experimental measure and type of cells under study, we find that GC4 content usually has a more positive effect on gene expression in unspliced genes relative to spliced ones (Figure 6, Table S1). In particular, unspliced mRNAs show a more positive/less negative correlation of GC4 with transcription initiation (GRO-cap data); cytoplasmic stability (exosome mutant); RNA (whole cell RNA-seq); cytoplasmic enrichment (cell fractionation), 
translation rate (ribosome profiling vs whole cell RNA-seq); and protein amount (mass-spec). These analyses suggest that GC4 content has an effect on the RNA abundance of intronless mRNA molecules, which is carried through to the protein expression. Taken together, these genome-wide analyses support our observation of a splicing-dependent relationship between codon usage and expression in human cells.

\section{Discussion}

We have shown that the effects of GC content on gene expression in human cells are splicing-dependent (the effect is larger in unspliced genes compared to spliced genes) and position-dependent (the effect is larger at the 5' end of genes than at the 3' end). In addition, human genes show striking patterns of codon usage, which differ between spliced and unspliced genes and between first and subsequent exons. Our results have implications for the understanding of the evolution of human genes and the functional consequences of synonymous codon usage.

\section{Mechanisms of splicing- and position-dependent effects of codon usage}

Specific patterns of codon usage have previously been found at the $5^{\prime}$ ends of genes in bacteria, yeast and other species (Gu et al., 2010; Kudla et al., 2009; Tuller et al., 2010). In bacteria and yeast, strong mRNA folding near the start codon prevents ribosome binding and reduces translation efficiency, resulting in selection against strongly folded 5' mRNA regions (Kudla et al., 2009; Shah et al., 2013). In addition a "ramp" of rare codons has been observed near the 5 ' end of RNAs in multiple species, with a possible role in preventing a wasteful accumulation of ribosomes on mRNAs (Tuller et al., 2010) or reducing the strength of mRNA folding (Bentele et al., 2013). These phenomena cannot explain our results in human, because both the folding energy and codon ramp models predict low GC content near the start codon, whereas we observe high GC content within first exons of human protein-coding genes (Figure 1B). Furthermore, our experiments show that high GC content near the start codon 
increases expression, whereas the folding energy and codon ramp models would predict low expression.

We propose instead that splicing- and position-dependent effects of GC content are explained by co-transcriptional or early post-transcriptional events in the lifetime of an mRNA. Using matched reporter gene libraries, we show that most, but not all, variants show an increase in expression when spliced. Splicing typically increases the expression of AT-rich variants, but it does not further increase the expression of GC-rich transcripts, which suggests that splicing and high GC content influence expression through at least one common mechanism. Splicing increases transcription (Kwek et al., 2002), prevents nuclear degradation (Nott et al., 2003), facilitates nuclear-cytoplasmic mRNA export through the Aly/REF-TREX pathway (Muller-McNicoll et al., 2016), and stimulates translation (Nott et al., 2004). High GC content might increase RNA polymerase processivity (Bauer et al., 2010; Zhou et al., 2016); GC-rich variants are less likely to contain cryptic polyadenylation sites (consensus sequence: AAUAAA) or destabilizing AU-Rich Elements (AREs) (Higgs et al., 1983); and high GC content near the 5' end may also facilitate cytoplasmic localisation of mRNA. GC-rich sequence elements of endogenous unspliced genes were previously shown to route transcripts into the splicing-independent ALREX nuclear export pathway, allowing efficient cytoplasmic accumulation (Palazzo et al., 2007). In agreement with this, low expression caused by inhibitory sequence features (such as low GC-content) can be rescued by extending the mRNA at the 5'end with a GC-rich sequence (Figure 3D,E, Figure S5). This may act as a compensatory mechanism when gene expression cannot rely on the positive regulatory effects of splicing (Palazzo and Akef, 2012). In contrast, it was recently shown that binding of HNRNPK to the GC-rich SIRLOIN motif leads to nuclear enrichment of lncRNAs (and also some mRNAs) (Lubelsky and Ulitsky, 2018). Our genomic analyses of IncRNA sequences do not show the same splicing-dependent compositional patterns as observed in mRNAs and it is therefore likely that antagonistic pathways act simultaneously in shaping the RNA expression landscape. Thus, we propose that the genomic patterns and their 
consequences on gene expression reported here are general features of proteincoding genes.

Recent studies also highlight patterns of codon usage as major determinants of RNA stability in yeast (Presnyak et al., 2015), zebrafish (Mishima and Tomari, 2016) and other species (Bazzini et al., 2016). The usage of less common, 'nonoptimal' codons within transcripts was shown to control poly-A tail length and RNA half-life in a translation-dependent manner through the coupled activity of different CCR4-NOT nucleases (Radhakrishnan et al., 2016; Webster et al., 2018). Consistent with these findings, we observed that CAI is positively correlated with mRNA expression levels in human cells. However, it remains to be seen whether the correlation of CAI with mRNA expression depends on translation. Because of the strong correlation between GC content and CAI, it is difficult to disentangle independent contributions of these variables. Additionally, we find that the correlation between GC content (or CAI) and expression is position- and splicingdependent, whereas no evidence for such context-dependence has been reported for the CCR4-NOT-mediated mechanism.

Other instances in which the effects of codon usage are context-dependent have been described. Most notably, tRNA populations and transcriptome codon usage patterns were shown to differ between mammalian tissues (Dittmar et al., 2006; Gingold et al., 2014; Plotkin et al., 2004; Rudolph et al., 2016). Intriguingly, genes preferentially expressed in proliferating cells and tissue-specific genes tend to be AT-rich, whereas genes expressed in differentiated cell types and housekeeping genes are more GC-rich (Gingold et al., 2014; Vinogradov, 2003). Although these differences have been interpreted in terms of the match between codon usage and cellular tRNA pools, it is plausible that translation-independent mechanisms contribute to context-dependent effects of codon usage. Accordingly, in Drosophila, codon optimality determines mRNA stability in whole cell embryos, but not in the nervous system, independent of tRNA abundance (Burow et al., 2018). Recently, it was shown that Zinc-finger Antiviral Protein (ZAP) selectively recognises high $\mathrm{CpG}$-containing viral transcripts as a mechanism to distinguish self from non-self (Takata et al., 2017). We speculate that similar regulatory 
proteins and mechanisms exist for cellular expressed genes. The cell lines used in the present study, HeLa and HEK293, are both rapidly proliferating and experimental results are correlated ( $\mathrm{r}=0.36$, Flow-Seq data), but divergent expression of some GFP variants was also observed. Similarly, the effect size of GC content on the expression of endogenously expressed genes varies with cell type. It would be interesting to compare the expression of our variants in other cell types to further address the question of tissue-specific codon usage and adaptation to tRNA pools.

\section{Implications for the evolution of protein-coding genes}

The fact that long, multi-exon genes are often found in GC-poor regions of the genome might result from regional mutation bias. However, an alternative explanation is possible: GC-poor genes may be under selective pressure to retain their introns, and intronless genes may experience selective pressure to increase their GC content. These possibilities are supported by multiple observations: Firstly, endogenous intronless genes are on average more GC-rich than introncontaining genes. Secondly, the GC content of functional (but not non-functional) retrogenes is higher compared to their respective intron-containing parental genes, which cannot be explained by a systematic integration bias. Thirdly, in genome-wide analysis, correlations between GC-content and expression are generally more positive (or less negative) for unspliced compared to spliced genes. Taken together, this suggests that for the long-term success of an unspliced gene (i.e. stable conservation of expression and functionality) an increase in GC content is essential. By contrast, splicing allows genes to remain functional even when mutation bias or other mechanisms lead to a decrease of their GC content. 


\section{Materials and Methods}

\section{Genes and plasmids}

412 The library of 217 synonymous GFP variants used here consists of 138 variants

413 from an earlier study (Kudla et al., 2009), 59 new variants assembled using the 414 same PCR-based method as in (Kudla et al., 2009), and 22 variants that were 415 designed in silico and ordered as synthetic gene fragments (gBlocks) from 416 Integrated DNA Technologies (IDT) (Mittal et al., 2018). Each of the 22 variants 417 was designed by setting a target GC3 content (between 25 and 95\%) and 418 randomly replacing each codon with one of its synonymous codons, such that the 419 expected GC3 content at each codon position corresponded to the target GC3 420 content. For example, to design a GFP variant with GC3 content of $25 \%$, each 421 glycine codon was replaced with one of the four synonymous glycine codons 422 with the following probabilities: GGA, 37.5\%; GGC, 12.5\%, GGG, 12.5\%; GGT, $42337.5 \%$. We also generated 23 mKate2 sequences using an analogous procedure 424 and ordered the variants as gBlocks from IDT. All the genes were cloned into the 425 Gateway Entry vector pGK3 (Kudla et al., 2009).

\section{Construction of transient expression vectors}

428 Plasmids used in transient transfection experiments are based on pCI-neo 429 (Promega), a CMV-driven mammalian expression vector that contains a chimeric 430 intron upstream of the multiple cloning site (MCS) within the 5'UTR. This intron 431 consists of the 5' splice donor site from the first intron of the human beta-globin 432 gene and the branch and 3' splice acceptor site from the intron of 433 immunoglobulin gene heavy chain variable region (see pCI-neo vector technical 434 bulletin, Promega). This vector was adapted to be compatible with Gateway 435 recombination cloning by inserting the Gateway-destination cassette, RfA, using 436 the unique EcoRV and SmaI restriction sites present within the MCS of pCI-neo, 437 generating pCM2. This plasmid was then further modified by removing the 438 intron contained within the 5'UTR by site-directed deletion mutagenesis using 439 Phusion-Taq (ThermoScientific) and primers 'pCI_del_F' and 'pCI_del_R' (see 440 Supplementary Table 2 for list of all primers used), generating plasmid pCM1. 
441 To be able to normalise spectrophotometric measurements from single GFP

442 transfection experiments, pCM1 and pCM2 were further modified to contain a

443 separate expression cassette driving the expression of a second fluorescent 444 reporter gene, mKate2. The mKate2 gene cassette from pmKate2-N (Evrogen) 445 was inserted via Gibson assembly cloning: First, the entire mKate2 expression 446 cassette was amplified using primers 'mKate2_gibs_F' and 'mKate2_gibs_R' which 447 add overhangs homologous to the pCM insertion site. Next, pCM1 and pCM2 448 were linearised by PCR using primers 'pCI_gib_F' and 'pCI_gib_R'. All PCR 449 products were purified using the Qiagen PCR purification kit and fragments with 450 homologous sites recombined using the Gibson assembly cloning kit (NEB) 451 according to manufacturer's instructions (NEB). Successful integration was 452 validated by Sanger sequencing. This generated plasmids pCM3 (-intron, $453+$ mKate2) and pCM4 (+intron, +mKate2).

\section{Transient plasmid transfections for spectrofluorometric measurements}

Plasmids for transient expression of fluorescent genes were transfected into HeLa cells grown in 96-well plates. Per plasmid construct, 3 technical replicates were tested by reverse transfection. Enough transfection mix for 4 wells was prepared by diluting 280ng plasmid DNA in 40ul OptiMem (Gibco). 1ul

460 Lipofectamine2000 (Invitrogen; 0.25ul per well) was diluted in 40ul OptiMem 461 and incubated for $5 \mathrm{~min}$ at room temperature. Both plasmid and 462 Lipofectamine2000 dilutions were then mixed (80ul total volume) and further 463 incubated for $20-30 \mathrm{~min}$. 20ul of transfection complex was then pipetted into 3 464 wells before adding 200ul of HeLa cell suspension (45,000 cells/ml; 9,000 465 cells/well) in phenol red-free DMEM (Biochrom, F0475). Media was exchanged $4663-4 \mathrm{~h}$ post-transfection to reduce toxicity. Cells were then grown for a further $24 \mathrm{~h}$ 467 or $48 \mathrm{~h}$ at $37 \mathrm{C}, 5 \% \mathrm{CO} 2$.

468 After incubation, cells were lysed by removing media and adding $200 \mathrm{ul}$ of cell 469 lysis buffer (25mM Tris, pH 7.4, 150mM NaCl, 1\% Triton X-100, 1mM EDTA, pH 470 8). Fluorescence readings were obtained using a Tecan Infinite M200pro 471 multimode plate reader. The plate was first incubated under gentle shaking for 472 15min followed by fluorescence measurements using the following settings: 
473 Ex486nm/Em 515nm for GFP and Ex588nm/Em633nm for mKate2; reading

474 mode: bottom; number of reads: 10 per well; gain: optimal.

475 For data analysis, measurements of untransfected cells were subtracted as

476 background from all other wells. For comparability of different plates within a

477 set of experiments, the same 3 genes were transfected on every plate to account

478 for technical variability. In the screen of individual GFP variants (see Figure 2),

479 GFP measurements were divided by mKate2 measurements from same wells to

480 reduce noise caused by well-to-well variation in transfection efficiency, but

481 similar results were obtained without normalisation.

\section{Transient transfections and RNA extraction for qRT-PCR analysis}

484 HeLa cells were reverse transfected in 12-well plates using 800ng plasmid DNA 485 and 2ul Lipofectamine 2000 (Invitrogen). DNA and Lipofectamine 2000 were 486 diluted in 100ul OptiMEM (Gibco) each, incubated for $5 \mathrm{~min}$, mixed and further 487 incubated for $20 \mathrm{~min}$. The transfection complex was then added to each well 488 before adding $10^{5} \mathrm{HeLa}$ cells. Cells were incubated for $24 \mathrm{~h}$ at $37 \mathrm{C}, 5 \% \mathrm{CO} 2$ before 489 harvesting. Cells were then harvested by adding $1 \mathrm{ml}$ Trizol reagent (Life 490 technologies). RNA was extracted according to manufacturer's instructions. 491 Resulting RNA was further treated with DNAse I using the Turbo DNase kit 492 (Ambion) to remove any residual plasmid and genomic DNA.

\section{qRT-PCR analysis}

495 cDNA for qRT-PCR analysis was prepared using SuperScript III Reverse 496 Transcriptase (Life technologies) according to the manufacturer's 497 recommendations with 500ng total RNA as template and 500ng random 498 hexamers (Promega). All qRT-PCRs were carried out on a Roche LightCycler 480 499 using Roche LightCycler480 SYBR Green I Master Mix and 0.3uM gene-specific 500 primers. Samples were analysed in triplicate as $20 \mathrm{ul}$ reactions, using $2 \mathrm{ul}$ of 501 diluted cDNA. Cycling settings: DNA was first denatured for $5 \mathrm{~min}$ at $95^{\circ} \mathrm{C}$ before 502 entering a cycle (50-60x) of denaturing for $10 \mathrm{sec}$ at $95^{\circ} \mathrm{C}$, annealing for $7 \mathrm{sec}$ at $50355-60^{\circ} \mathrm{C}$ (depending on primers used), extension for $10 \mathrm{sec}$ at $72^{\circ} \mathrm{C}$ and data 504 acquisition. DNA was then gradually heated up by $2.20^{\circ} \mathrm{C} / \mathrm{s}$ from 65 to $95^{\circ} \mathrm{C}$ for 5055 sec each and data continuously collected (Melting curve analysis). Data was 


\section{1}

512

evaluated using the comparative Ct method (Livak and Schmittgen, 2001). RNA measurements from transient transfection experiments were normalised to the abundance of neomycin RNA, which is expressed from the same plasmid, to control for differences in transfection efficiency (primers 'Neo_F' and 'Neo_R').

\section{Subcellular fractionation}

This protocol is based on the cellular fractionation protocol published by (Gagnon et al., 2014) but includes a further clean-up step using a sucrose cushion as described by (Zaghlool et al., 2013) and a second lysis step as described by (Wang et al., 2006). Cell lysis and nuclear integrity was monitored throughout by light microscopy following Trypan blue staining (Sigma). Cells were grown in $10 \mathrm{~cm}$ plates for $24 \mathrm{~h}$ to about $90 \%$ confluency. Cells were then washed with PBS and trypsinised briefly using $1 \mathrm{ml}$ of $1 x$ Trypsin/EDTA. After stopping the reaction with $5 \mathrm{ml}$ DMEM, cells were transferred into $15 \mathrm{ml}$ falcon tubes and collected by spinning at $100 \mathrm{~g}$ for $5 \mathrm{~min}$. Resulting cell pellets were resuspended in $500 \mathrm{ul}$ icecold PBS, transferred into $1.5 \mathrm{ml}$ reaction tubes and spun at $500 \mathrm{~g}$ for $5 \mathrm{~min}, 4^{\circ} \mathrm{C}$. The supernatant was discarded and cells resuspended in 250ul HLB (10mM Tris (pH 7.5), $10 \mathrm{mM} \mathrm{NaCl}, 3 \mathrm{mM} \mathrm{MgCl} 2,0.5 \%(\mathrm{v} / \mathrm{v}) \mathrm{NP} 40,10 \%(\mathrm{v} / \mathrm{v})$ Glycerol, $0.32 \mathrm{M}$ sucrose) containing 10\% RNase inhibitors (RNasin Plus, Life Tech) by gently vortexing. Samples were then incubated on ice for $10 \mathrm{~min}$. After incubation, samples were vortexed gently, spun at $1000 \mathrm{~g}$ for $3 \mathrm{~min}, 4^{\circ} \mathrm{C}$, and supernatants and pellets were processed separately as indicated in a) and b) below.

a) Cytoplasmic extract:

The supernatant was carefully layered over $250 \mathrm{ul}$ of a $1.6 \mathrm{M}$ sucrose cushion and spun at $21,000 \mathrm{~g}$ for $5 \mathrm{~min}$. The supernatant was then transferred into a fresh $1.5 \mathrm{ml}$ tube and $1 \mathrm{ml}$ Trizol was added and mixed by vortexing.

b) Nuclear extract:

The pellets were washed 3 times with HLB containing RNase inhibitors by gently pipetting up and down 10 times followed by a spin at $300 \mathrm{~g}$ for $2 \mathrm{~min}$. After the 3rd wash, nuclei were resuspended in 250ul HLB and 25ul (10\%) of detergent mix (3.3\% (wt/wt) sodium deoxycholate/6.6\% (vol/vol) Tween 40) dropwise added while vortexing slowly (600 rpm). Nuclei were then incubated for $5 \mathrm{~min}$ on ice before spinning at $500 \mathrm{~g}$ for $2 \mathrm{~min}$. The supernatant was discarded and pellets 
resuspended in $1 \mathrm{ml}$ Trizol (Ambion) by vortexing. 10ul 0.5M EDTA are added to each nuclear sample in Trizol and tubes heated to $65^{\circ} \mathrm{C}$ for $10 \mathrm{~min}$ to disrupt very strong Protein-RNA and DNA-RNA interactions. Tubes were then left to reach room temperature and RNA was extracted following the manufacturer's instructions.

\section{Transcription inhibition assay}

HeLa T-Rex Flp-in cell lines were grown to 80-90\% confluency in 6 well for $24 \mathrm{~h}$ before treatment with 500nM Triptolide (Sigma). Cells were harvested at indicated time points and RNA extracted using Trizol reagent (Ambion). Control cells were treated with the equal volume of DMSO (drug carrier). To assess transcript levels, qRT-PCR was performed as described above. GFP levels were normalised to levels of 7SK, a RNA polymerase III-transcribed non-coding RNA, whose expression levels are not affected by Triptolide treatment. Relative transcript levels of c-Myc are shown as an example of a relatively unstable transcript.

\section{Generation of stable Flp-in cell lines}

We adopted a multiplex-Gateway integration method to create a pool of 217 GFP plasmids which are compatible with the T-Rex Flp-in system (Invitrogen) for creating stable, doxycycline-inducible cell lines, in which each variant is expressed from the same genomic locus, allowing direct comparison of expression levels.

pcDNA5/FRT/TO/DEST (Aleksandra Helwak, University of Edinburgh) contains the Gateway-compatible attB destination cassette to allow the subcloning of genes from any Gateway-entry vectors. This plasmid was further modified to contain the same 5'UTR intron sequence as in pCM4 used in transient expression experiments using Gibson Assembly (NEB): the intronic sequence was amplified from pCM4 by PCR using primers 'Gib_intr_F' and 'Gib_intr_R' using Q5 HighFidelity Polymerase (NEB). The primers added 15nt overhangs which are homologous to the ends of pcDNA5/FRT/TO/DEST when linearised with AflII. The Gibson assembly reaction was performed as per manufacturer's instructions (NEB), generating pcDNA5/FRT/TO/DEST/INT. 
217 individual GFP variants stored in Gateway-entry vector pGK3 were mixed with a concentration of 0.06 ng of each GFP variant. For each pcDNA5 destination vector, a separate Gateway LR reaction was set-up in a total volume of $45 \mathrm{ul}$ using 500ng destination vector, 5ul LR Clonase enzyme mix, 38ul of the mixed 217 pGK3-GFP plasmids and TE (pH 8). The reactions were incubated at 25C overnight followed by Proteinase K digest (5ul, LR Clonase kit) for 10min at 37C. The total 50ul reaction mix was transformed into $2.5 \mathrm{ml}$ highly competent DH5alpha in a $15 \mathrm{ml}$ Falcon tube by heat-shocking cells for $2 \mathrm{~min} 30 \mathrm{~s}$ at $42 \mathrm{C}$, followed by cooling on ice for $3 \mathrm{~min}$, before adding $10 \mathrm{ml}$ SOC medium and incubating while shaking for $1 \mathrm{~h}$ at $37 \mathrm{C}$. After incubation, cells were spun down at $3000 \mathrm{~g}$ for $3 \mathrm{~min}$ and resulting bacterial pellets resuspended in $1 \mathrm{ml}$ fresh SOC. 10x100ul were plated onto L-Ampicillin agar plates and incubated overnight at $37 \mathrm{C}$ resulting in $>800$ colonies per plate. Bacterial colonies were scraped off the plates and collected in a falcon tube. Plasmid DNA was extracted using a Qiagen Midiprep kit according to the manufacturer's instructions, resulting in two plasmid pools: pCDNA5/GFPpool and pcDNA5/INT/GFPpool. Both pools were subjected to high-throughput sequencing to confirm the presence of different GFP variants.

HeLa T-Rex Flp-in cells (gifted by the Andrew Jackson lab, The University of Edinburgh) and Hek293 T-Rex Flp-in (Thermo Scientific) were grown to 80\% confluency in 6 well plates. For GFP plasmid pool transfections, pCDNA5/GFPpool or pCDNA5/INT/GFPpool were mixed in a 9:1 ratio with the Flp-recombinase expression plasmid p0G44 (Invitrogen) to give 2ug in total (1.8ug pOG44 + 0.2ug pCDNA5) and diluted in OptiMEM (Gibco) to 100ul. Transfections were performed with 9ul Lipofectamine2000 (Invitrogen) and $91 \mathrm{ul}$ OptiMEM per well by incubating $5 \mathrm{~min}$ at room temperature before mixing with plasmid DNA and a further 15min incubation. The transfection mix was then added dropwise to the cells. Media were replaced with conditioned media 4h post-transfection. Cells were incubated for further $48 \mathrm{~h}$ before chemical selection to select for successful gene integration using 10ng/ul Blasticidin S (ThermoFisher) and 400mg/ml (HeLa T-Rex Flp-in) or 100mg/ml (Hek293 TRex Flp-in) Hygromycin B (Life Technologies). Successful selection was determined by monitoring cell death in untransfected cells. Chemically resistant 
605

606

607

608

609

610

611

612

613

614

615

616

617

618

619

620

621

622

623

624

625

626

627

628

629

630

631

632

633

634

635

636

637

cells represent pools of cell lines expressing different GFP variants from the same genomic locus. High-throughput sequencing of the GFP integration site within each generated cell line pool confirmed the successful integration of all variants.

HeLa T-Rex Flp-in and Hek293 T-Rex Flp-in cell lines expressing two individual GFP variants (GC3=96\% and GC3=36\%; see Supplementary Figure 3) as spliced and unspliced transcripts were generated using the same protocol.

\section{Flow-Seq: FACS sorting and genomic DNA extraction}

80x15cm cell culture plates of HeLa T-Rex Flp-in GFP pool cells and 40x15cm cell culture plates of Hek293 T-Rex Flp-in GFP pool cells were induced with 1ug/ml Doxycyline (Sigma, D9891) in phenol red-free DMEM (Biochrom, F0475) supplemented with 10\% FCS (Sigma, F-7524) and 2mM L-Glutamine. After 24h or $48 \mathrm{~h}$, cells were harvested by gentle trypsinisation and cells were sorted into 8 fluorescence bins using a BD FACS Aria II cell sorter. To define the range of GFP positive signal, cells without stable GFP expression were used as negative control. 80\% of HeLa and 90\% Hek293 GFP pool cells fell into the GFP-positive range. Each fluorescence bin was chosen to comprise roughly $10 \%$ of the GFPpositive population. The bin spacing was kept the same for the sorting of HeLa cell pools expressing unspliced and spliced GFP variants to allow direct comparisons of the fluorescence profiles of individual variants.

About $10^{7}$ cells per bin were collected in Polypropylene collection tubes (Falcon) coated with 1\% BSA/PBS, cushioned with 200ul 20\%FBS/PBS. Cell suspensions were decanted into $15 \mathrm{ml}$ tubes and cells collected by spinning $5 \mathrm{~min}$ at $500 \mathrm{~g}$. The supernatant was transferred into fresh $15 \mathrm{ml}$ tubes and precipitated using 2 volumes of 100\% EtOH/0.1 volume Sodium Acetate (pH 5.3) and 10ul Glycoblue (Ambion). Tubes were shaken vigorously for 10 s before incubating at $-20 \mathrm{C}$ for $15 \mathrm{~min}$, followed by spinning at $3000 \mathrm{~g}$ for $20 \mathrm{~min}$. Resulting pellets were airdried, resuspended in $1 \mathrm{ml}$ digest buffer (100mM Tris pH 8.5, 5mM EDTA, 0.2\% SDS, $200 \mathrm{mM} \mathrm{NaCl}$ ) and then combined with the respective cell pellet. 10ul RNAse A (Qiagen, 70U) was added and samples gently rotated at 37C. After $1 \mathrm{~h}, 1 \mathrm{ul} / \mathrm{ml}$ Proteinase $\mathrm{K}(20 \mathrm{mg} / \mathrm{ml}$, Roche) was added to the samples before rotating a further $2 \mathrm{~h}$ at 55C. Genomic DNA was purified 3 times by using 1 volume 
638 Phenol:Chloroform:Isoamyl alcohol (PCI, 25:24:1, Sigma). After each addition of

639 PCI, samples were shaken vigorously for $10 \mathrm{~s}$ before spinning at $3000 \mathrm{~g}$ for $20 \mathrm{~min}$

640 (first extraction) or $5 \mathrm{~min}$ (all following). The resulting bottom layers including

641 the interphase were removed before each PCI addition. After the last PCI

642 extraction, the upper layer was transferred into a fresh $15 \mathrm{ml}$ tube and 1

643 extraction performed using 1 volume chloroform:isoamyl alcohol (CI,24:1,

644 Sigma). After a $5 \mathrm{~min}$ spin at $3000 \mathrm{~g}$, the upper layer was transferred into a fresh

$64515 \mathrm{ml}$ tube and DNA precipitated using EtOH/Sodium Acetate as before. After a

$6465 \mathrm{~min}$ incubation on ice, DNA was collected by spinning for $30 \mathrm{~min}$ at $3000 \mathrm{~g}$. The

647 resulting DNA pellets were washed 2 times with 75\% EtOH before air-drying and

648 resuspending in $200 \mathrm{ul}$ Tris-EDTA $(10 \mathrm{mM})$. The quality of the extracted genomic

649 DNA was assessed on a $0.8 \%$ Agarose/TBE gel.

650

651 Polysome profiling

652 Hek293 Flp-in GFP pool cell lines were grown to $90 \%$ confluency on $15 \mathrm{~cm}$

653 dishes. Cells were treated for $20 \mathrm{~min}$ with 100ug/ul Cycloheximide before

654 harvesting cells by removing media, washing with 2x ice-cold PBS followed by

655 scraping cells into $1 \mathrm{ml}$ PBS and transferring into $1.5 \mathrm{ml}$ tubes. Cells were pelleted

656 at $7000 \mathrm{rpm}, 4^{\circ} \mathrm{C}$ for $1 \mathrm{~min}$ and resulting cell pellet carefully resuspended by

657 pipetting up and down in 250ul RSB (10x RSB: 200mM Tris (pH 7.5), $1 \mathrm{M} \mathrm{KCl,}$

658 100mM MgCl2) containing 1/40 RNasin (40U/ul, Promega), until no clumps

659 were visible. $250 \mathrm{ul}$ of polysome extraction buffer was then added (1ml 10x RSB

$660+50 \mathrm{ul} \mathrm{NP}-40$ (Sigma) + 9ml H2O + 1 complete mini EDTA-free protease inhibitor

661 pill (Roche)) and lysate passed 5x through a 25G needle avoiding bubble

662 formation. The lysate was then incubated on ice for $10 \mathrm{~min}$ before spinning

$66310 \mathrm{~min}$ at $10,000 \mathrm{~g}, 4^{\circ} \mathrm{C}$. The supernatant was then transferred into a fresh $1.5 \mathrm{ml}$

664 tube and the RNA concentration estimated by measuring the OD at $260 \mathrm{~nm}$.

665 Sucrose gradients (10-45\%) containing $20 \mathrm{mM}$ Tris, $\mathrm{pH}$ 7.5, $10 \mathrm{mM} \mathrm{MgCl2}$, and

$666100 \mathrm{mM} \mathrm{KCl}$ were made using the BioComp gradient master. 100ug of Lysate

667 were loaded on sucrose gradients and spun at $41,000 \mathrm{rpm}$ for $2.5 \mathrm{~h}$ in a Sorvall

668 centrifuge with a SW41Ti rotor. Following centrifugation, gradients were

669 fractionated using a BioComp gradient station model 153 (BioComp 23 
670

671

672

673

674

675

676

677

678

679

680

681

682

683

684

685

686

687

688

689

690

691

692

693

694

695

696

697

698

699

700

701

Instruments, New Brunswick, Canada) by measuring cytosolic RNA at $254 \mathrm{~nm}$ and collecting 18 fractions.

RNA from all fractions was precipitated using 1 volume of $100 \% \mathrm{EtOH}$ and $1 \mathrm{ul}$ Glycoblue (Ambion), before extracting RNA using the Trizol method (Life Technologies). Equal volumes of RNA of each fraction was run on a $1.3 \%$ Agarose/TBE gel to assess the quality of fractionation and RNA integrity. Additionally, equal volumes of RNA of each fraction were used in cDNA synthesis using SuperScript III (ThermoFisher) and 2uM gene-specific primers for GFP ('pcDNA5-UTR_R') and GAPDH ('GAPDH_R') followed by qRT-PCR analysis. For high-throughput sequencing, total RNA from collected fractions was combined in equal volumes into 4 pools (as indicated in Figure 5B; free ribonucleoprotein (RNP) complexes, monosomes, light polysomes (2-4) and heavy polysomes (5+)) before amplicon library preparation (as described below).

\section{High-throughput library preparation and sequencing}

Sequencing libraries were generated by PCR using primers specific for GFP amplification (Supplementary Table 2) which carry the required adaptor sequences for paired-end MiSeq sequencing, as well as $6 \mathrm{nt}$ indices for library multiplexing. Between 6-10ug of total genomic DNA were used in multiple PCR reactions (200ng per 50ul reaction). All PCRs were performed using Accuprime Pfx (NEB) according to manufacturer's recommendations using 0.4ul Accuprime Pfx Polymerase and 0.3uM of each primer ('PE_PCR_left' and 'S_indexX_right_PEPCR'). The cycling conditions were as follows: Initial denaturation at $95 \mathrm{C}$ for $2 \mathrm{~min}$, followed by 30 cycles of denaturation at $95 \mathrm{C}$ for $15 \mathrm{sec}$, annealing at $51 \mathrm{C}$ for $30 \mathrm{sec}$, extension at $68 \mathrm{C}$ for $1 \mathrm{~min}$. The final extension was performed at $68 \mathrm{C}$ for $2 \mathrm{~min}$. After PCR, all reactions of the same template were pooled and 1/3 of the reaction purified using the Qiagen PCR purification kit according to the manufacturer's instructions. DNA was eluted in 50ul H2O. Library size selection was performed using the Invitrogen E-gel system (Clonewell gels, 0.8\% agarose) followed by Qiagen MinElute PCR purification. Correct fragment sizes were confirmed and quantified using the Agilent Bioanalyzer 2100 system. 
702 For library preparation of RNA samples, 500ng RNA was first converted into

703 cDNA using 2nmol GFP-specific primers ('S_indexX_right_PEPCR') using

704 SuperScript III (Life technologies) according to manufacturer's protocol, using

$70550 \mathrm{C}$ as extension temperature. Resulting cDNA was then treated with $1 \mathrm{ul}$

706 RNaseH (NEB) for $20 \mathrm{~min}$ at $37 \mathrm{C}$, followed by heat inactivation at $65 \mathrm{C}$ for $5 \mathrm{~min}$.

707 Samples were diluted 1:2.5 before using 2ul as template in PCR for library

708 preparation. A minimum of $8 \times 50 \mathrm{ul}$ PCR reactions were set up and pooled for

709 each sample before PCR purification, followed by E-gel purification as described 710 above.

711 High-throughput sequencing was conducted by Edinburgh Genomics (The 712 University of Edinburgh) and Imperial BRC Genomics facility (Imperial College

713 London) using the Illumina MiSeq platform (2x300nt paired-end reads).

715 Analysis of GFP pool experiments

716 Raw sequencing files (fastq files) were demultiplexed by $6 \mathrm{nt}$ indices by the 717 respective sequencing facility. To remove the plasmid sequence, the second 718 reads from paired-end sequencing were trimmed using flexbar (-as 719 ATGTGCAGGGCCGCGAATTCTTA -ao 4 -m 15 -u 30). Reads were then mapped to 720 the GFP library using bowtie2 (-X 750) and filtered using samtools (-f 99).

721 For Flow-seq data, only variants with a minimum of 1000 reads across all 8 722 sequencing bins were used for further analysis. For each GFP variant, the 723 number of reads in each bin (n(i)) was multiplied by the respective bin index (i)

724 before taking the sum and dividing by the total number of reads across all bins:

725 Fluorescence (variant) $=\sum_{i=1}^{8} i * n(i) / \sum_{i=1}^{8} n(i)$

726 For cell fractionation experiments, only data with a minimum of 1000 reads

727 across both cytoplasmic and nuclear fractions was used to calculate the relative 728 cytoplasmic concentration ('RCC') for each variant: $R C C=\frac{n(\text { cyto })}{n(c y t o)+n(n u c)}$

729 For polysome profiling, only variants with a minimum of 1000 reads across all 4 730 sequencing bins were used for further analysis. To estimate ribosome density, 731 for each GFP variant, the number of reads in each bin (n(i)) was multiplied by the 732 respective polysomal fraction index (i) before taking the sum and dividing by the 733 total sum of reads across all fractions: 
734 Ribosome density(variant $)=\sum_{i=1}^{4} i * n(i) / \sum_{i=1}^{4} n(i)$

735 Ribosome association for each variant was calculated as the sum of reads (n) in

736 light polysomes, heavy polysomes and monosomal fractions, divided by the sum

737 of reads found in the free RNP fraction:

738 Ribosome association(variant $)=(\mathrm{n}$ (monosomes $)+\mathrm{n}$ (light polysomes $)+$

$739 \mathrm{n}$ (heavy polysomes)) / n(free RNPs)

\section{Definition of calculated sequence features}

742 GC3: GC content in the third position of codons

743 CpG: number of $\mathrm{CpG}$ dinucleotides

$744 \mathrm{dG}$ : The minimum free energy of predicted mRNA secondary structure around

745 the start codon was calculated using the hybrid-ss-min program version 3.8

746 (default settings: $\mathrm{NA}=\mathrm{RNA}, \mathrm{t}=37,[\mathrm{Na}+]=1,[\mathrm{Mg}++]=0$, maxloop $=30$, prefilter

$747=2 / 2$ ) in the 42-nt window (-4 to 38) as in (Kudla et al., 2009).

748 CAI: Codon Adaptation Index (H. sapiens) (Sharp and Li, 1987a) was calculated

749 using a reference list of highly expressed human genes collected from the EMBL-

750 EBI expression atlas https://www.ebi.ac.uk/gxa.

751 tAI: tRNA adaptation index (dos Reis et al., 2004)

752 ARE: top score of ATTTA motif match in each sequence.

753 AT-stretch: number of times motif (AT) $\{9\}$ was identified in each sequence.

754 GC-stretch: number of times motif (GC) $\{9\}$ was identified in each sequence.

755 Poly_A: number of times the position-specific scoring matrix $756((47,3,0,50)(18,6,9,67)(53,12,12,23)(59,6,0,35)(70,6,6,18))$ was identified in 757 each sequence.

758 SD_cryptic: number of times RSGTNNHT motif was identified in each sequence.

759 SD_PSSM: number of times the position-specific scoring matrix $760 \quad((60,13,13,14)(9,3,80,7)(0,0,100,0)(0,0,0,100)(53,3,42,3)(71,8,12,9)(7,6,81,6)(1$ $7616,17,21,46)$ ) was identified in each sequence.

763 FIMO (http://meme-suite.org) was calculated to identify and count sequence 764 motifs. Open-source packages available for $\mathrm{R}$ were used for generating 765 correlation matrices (corrplot), heatmaps (ggplot2), boxplots 
766 (graphics/ggplot2), The GC3 of all human coding sequences (assembly:

767 GRCg38_hg38; only CDS exons) was calculated using R package 'seqinr'.

768

769

\section{Computation methods for analysis of endogenous gene expression}

\section{Data Collection - see also Supplementary Table 1}

1. GC4 content was calculated for each protein-coding transcript annotated in GENCODE version 19 as the GC content of the third codon position across all fourfold-degenerate codons $\left(\mathrm{CT}^{*}, \mathrm{GT}^{*}, \mathrm{TC}^{*}, \mathrm{CC}^{*}, \mathrm{AC}^{*}, \mathrm{GC}^{*}, \mathrm{GA}^{*}\right.$, $\mathrm{CC}^{*}, \mathrm{GC}^{*}$ ). The core promoter of each transcript is further defined as -300 $\mathrm{bp} /+100 \mathrm{bp}$ around the annotated TSS.

2. The level of transcription initiation was quantified in K562 and Gm12878 cells as the number of GRO-cap reads from the same strand which overlap the core promoter.

3. Nuclear stability was assessed using CAGE data obtained in triplicate from Egfp, Mtr4 and Rrp40 knockdowns (GSE62047; (Andersson et al., 2014)). Similarly to the approach used for the GRO-cap data, we calculated the RPKM across core promoters for each library separately. The baseMean expression for each treatment was quantified using DESeq2, where promoters with no reads across any replicate were first removed from each comparison. Nuclear stability was then assessed as the fold-change between the Egfp and Mtr4 knockdown and cytoplasmic stability by the estimated fold-change between the Mtr4 and Rrp40 knockdowns.

4. The level of the mature mRNA was quantified using RNA-seq libraries from whole cell samples (prepared as described elsewhere for HEK293 $\begin{array}{llll}\text { cells and fownloaded } & \text { from }\end{array}$ http://hgdownload.cse.ucsc.edu/goldenPath/hg19/encodeDCC/wgEncod eCshlLongRnaSeq for Gm12878, HepG2, HeLa, Huvec and K562 cells). Reads were pseudoaligned against GENCODE transcript models using Kallisto, set with 100 bootstraps. All other parameters were left at their default. Transcript expressions were extracted as the estimated TPM (tags per million) values. 
5. The level of the mature mRNA in the nuclear and cytoplasmic fractions was quantified using Kallisto as previously. As transcript stability was similar in both fractions (linear regression coefficient $0.97, \mathrm{p}<2.2 \times 10^{-16}$ ), nuclear export was determined as the fraction TPM from these two compartments which was present in the nuclear fraction.

6. Ribosome-sequencing data from HEK293 (GSE94460) and HeLa (GSE79664) cells were used to quantify the level of mRNA translation in these two cells. Both of these measures were determined at the gene level, and so these observations were applied to all GENCODE transcripts annotated to these associated genes. These data were normalised to the mean mRNA expression in the relevant cell types (from step 4).

7. Protein expression was assessed using mass-spectrometry data (Geiger et al., 2012) (Supp. Table 2) as the mean LFQ intensity across three replicates for each uniprot-annotated gene in each cell line for which data were available. Only data from genes where the UniProt ID is uniquely linked to a single transcript were considered in the analyses presented here.

8. Protein stability was calculated as the level of the mature protein in HEK293 and HeLa cells (step 7) relative to the mean rate of mRNA translation in these cells (step 6).

\section{Regression modelling}

A pseudocount of 0.0001 was added to each measurement of gene expression and, excluding the nuclear export data, these values were then log2-transformed to generate a normal distribution of expression for subsequent analysis. Transcripts with an expression value of 0 were removed from downstream analysis and the resulting distributions used for regression analysis are displayed in Supplementary Figure 6. Transcripts were separated into unspliced and spliced, where splicing was defined as containing more than one exon in the GENCODE transcript model. Expression measurements were then linearly regressed against the GC4 content separately for each class of transcript and the coefficients along with their associated standard errors. These data were then 
828

829

830

831

832

833

834

835

836

837

838

839

840

841

842

843

844

845

846

847

848

849

850

851

852

853

854

855

856

857

858

859

860

bootstrapped by sampling with replacement and recalculating the regression coefficients for spliced and unspliced transcripts. The 95\% confidence interval of these coefficients (discounting the standard error in these estimations) obtained by 1,000 samplings of this type was used to draw the ellipses shown in Figure 6.

\section{Analysis of GC content variation in the human genome}

The GRCh38 sequence of the human genome, as well as the corresponding gene annotations (Ensembl release 85), was retrieved from the Ensembl FTP site (Zerbino et al., 2018). The full coding sequences (CDSs) of protein-coding genes were extracted, filtered for quality and clustered into putative paralogous families (see (Savisaar and Hurst, 2016) for full details). For all analyses, a random member was picked from each putative paralogous cluster. In addition, only one transcript isoform (the longest) was considered from each gene. Note that exon rank was always counted from the first exon of the gene, even if it was not coding. For Figure 1C, GC4 was averaged across all sites that were at the same nucleotide distance to the TSS and within an exon of the same rank. For the functional retrocopies analysis, the parent-retrocopy genes derived in (Parmley et al., 2007) were used. Pseudogenic retrocopies were retrieved from RetrogeneDB (Rosikiewicz et al., 2017). Retrocopy annotations were filtered to only leave human genes with a one-to-one ortholog in Macaca mulatta. Next, only ortholog pairs where both the human and the macaque copy were annotated as not having an intact reading frame and where the human copy was annotated as KNOWN_PSEUDOGENE were retained. For the analyses reported in Supplementary Figure 1, the functional retrocopies were also retrieved from RetrogeneDB, as we could not access genomic locations for the (Parmley et al., 2007) set. The functional retrogenes were retrieved similarly to pseudogenes, except that both the human and the macaque copy were required to have an intact open reading frame and the human copy could not be annotated as KNOWN_PSEUDOGENE.

Python 3.4.2. was used for data processing and R 3.1.2 was used for statistics and plotting (R Development Core Team, 2005).

\section{Acknowledgments}


861 We thank Elisabeth Freyer for help with cell sorting; Andrew Jackson, Nick

862 Gilbert and Aleksandra Helwak for gifts of cell lines and plasmids; James Brindle

863 for technical assistance; Michael Liss and members of Kudla and Hurst groups for

864 discussions; Edinburgh Genomics (University of Edinburgh) and the Imperial

865 BRC Genomics facility for next-generation sequencing; and Institute of Genetics

866 and Molecular Medicine technical support for help with media preparation and

867 sequencing. This work was supported by the Wellcome Trust (Fellowships

868097383 and 207507 to G.K.), the European Research Council (Advanced grant

869 ERC-2014-ADG 669207 to L.D.H.), and the Medical Research Council (Grants

870 MC_UU_00007/11 to M.S.T. and MC_UU_00007/12 to G.K. and PhD studentship to

871 C.M.).

872

873 
A

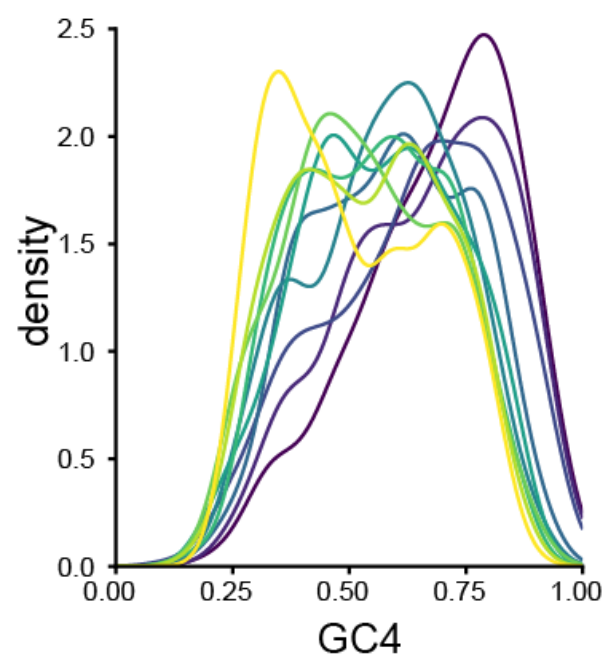

B

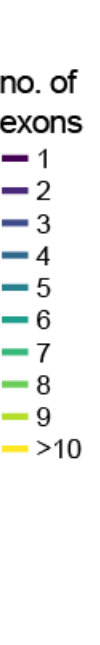

no. of exons

$-1$

$-2$

$-3$

$-4$

$-5$

$-6$

$-7$

$-8$

$-9$

$-10$

875

C

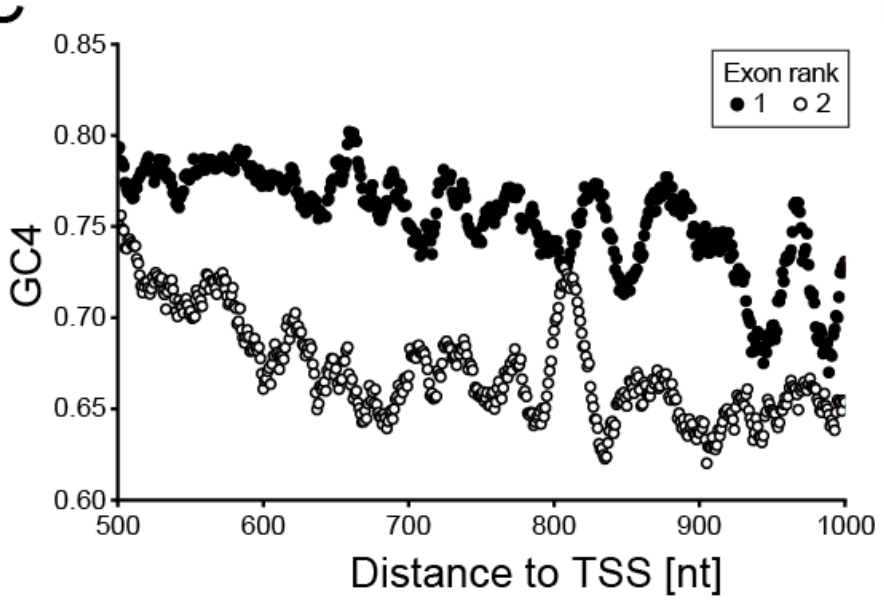

$\mathrm{D}$

exon rank

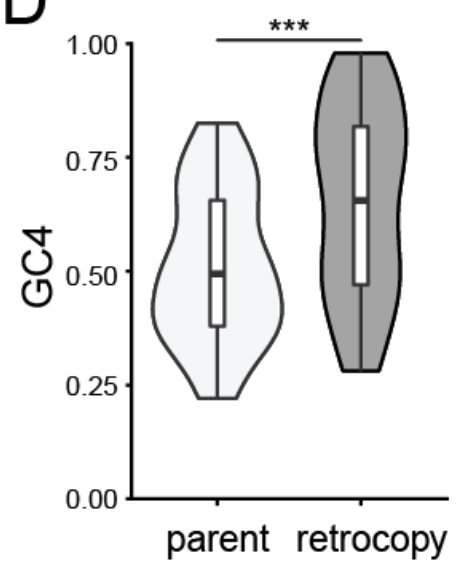

876

Figure 1. Splicing- and position-dependent patterns of nucleotide composition in human genes

879 (A) GC4 distribution of human protein-coding genes, grouped by number of 880 exons per gene.

881 (B) Mean GC4 content in protein-coding exons, grouped by exon position (rank) 882 and by number of exons per gene.

883 (C) Mean GC4 for individual codons within exons of rank 1 (black dots) or rank 2 884 (white dots) downstream of the transcription start site (TSS).

885 (D) GC4 distribution of functional retrogenes (dark grey) and their 886 corresponding parental genes (light grey) conserved between mouse and human $\left(p=2.1 \times 10^{-4}\right.$, from one-tailed Wilcoxon signed rank test, $\left.n=49\right)$. 

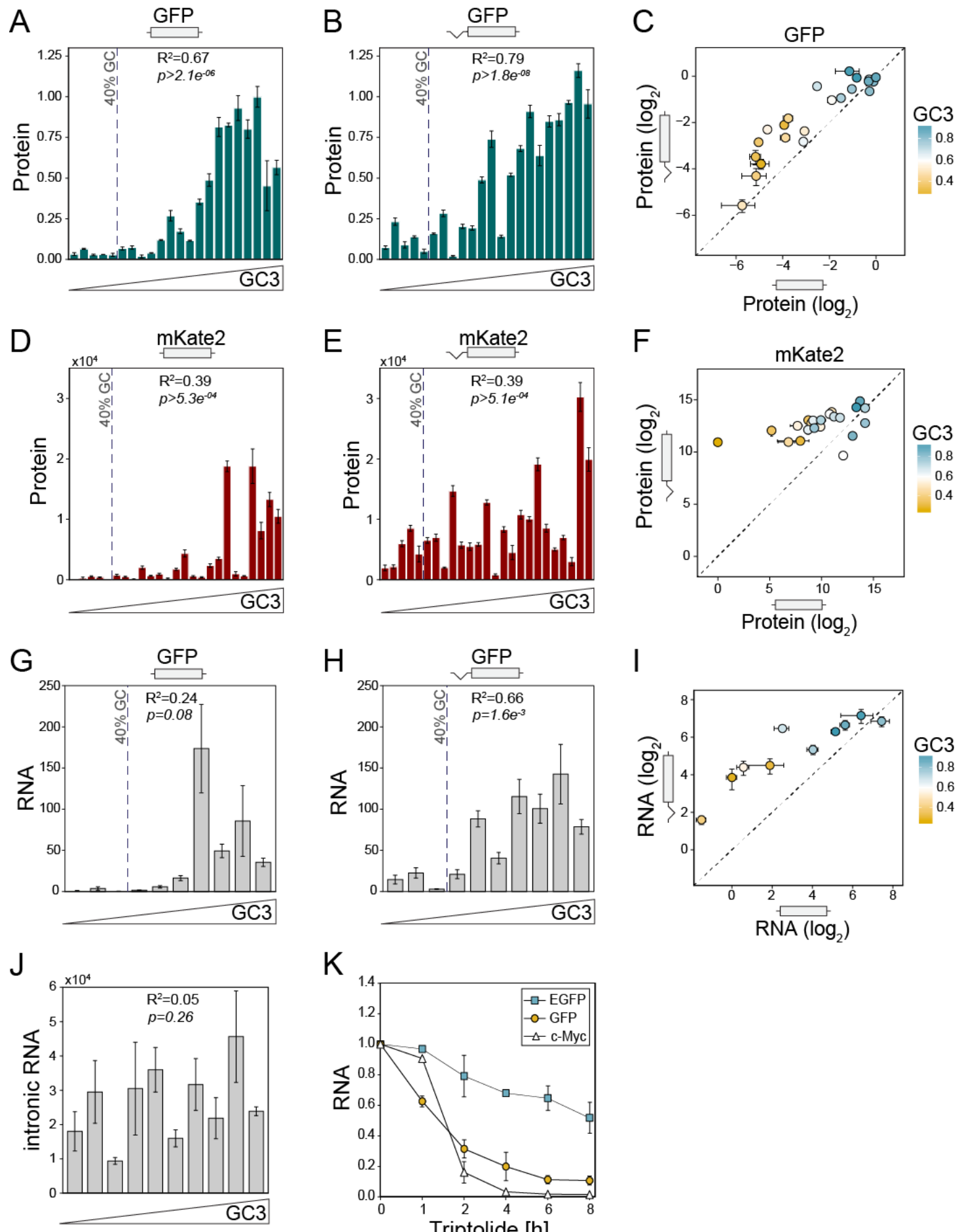

889

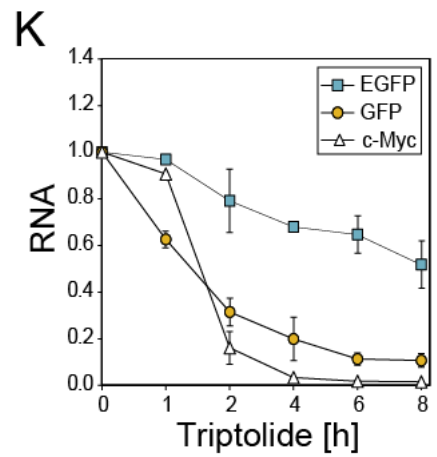

890

891 Figure 2. The effect of GC content on gene expression depends on splicing.

892 (A-B) Protein levels of 22 GFP variants when transiently expressed as unspliced

893 (A) or spliced (B) constructs were expressed in HeLa cells and quantified by 894 spectrofluorometry. Each data point represents the mean of 9 replicates, +/895 SEM. 
896 (C) Correlation of protein levels between unspliced and spliced variants of GFP

$897\left(\mathrm{n}=22, \mathrm{R}^{2}=0.69, \mathrm{p}=9.0 \times 10^{-7}\right)$. The dashed line indicates $\mathrm{x}=\mathrm{y}$.

898 (D-E) Protein levels of 23 mKate2 variants in the absence (A) or presence (B) of 899 splicing. Each data point represents the mean of 9 replicates.

900 (F) Correlation of protein levels between unspliced and spliced variants of 901 mKate2 $\left(\mathrm{n}=23, \mathrm{R}^{2}=0.29, \mathrm{p}=2.8 \times 10^{-4}\right)$.

902 (G-H) mRNA levels of 10 GFP variants when transiently expressed as unspliced

$903(\mathrm{G})$ or spliced $(\mathrm{H})$ constructs in HeLa cells and quantified by qRT-PCR. Data 904 points represent the mean of 3 replicates, +/- SEM.

905 (I) Comparison of mRNA expression from spliced and unspliced GFP variants.

906 (J) Intronic RNA levels of GFP variants measured by qRT-PCR.

907 (K) RNA stability time course of GC-rich (97\% GC3; $\left.t_{1 / 2}=8.6 \mathrm{~h}\right)$ and GC-poor (33\% 908 GC3, $\left.\mathrm{t}_{1 / 2}=2.4 \mathrm{~h}\right)$ GFP variants in stably transfected HeLa Flp-In cells after blocking 909 transcription with 500nM Triptolide. Results represent the averages of 2 910 independent experiments, +/- SD. 
A

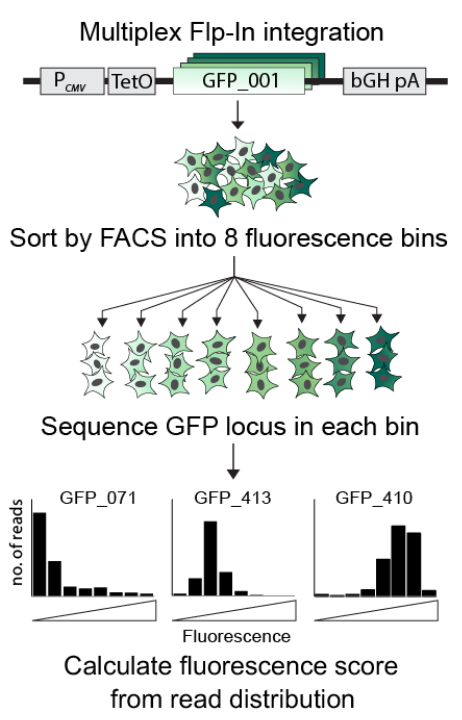

C

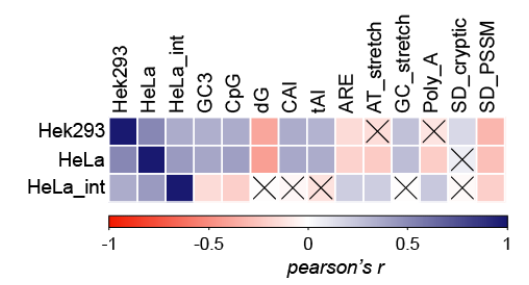

D

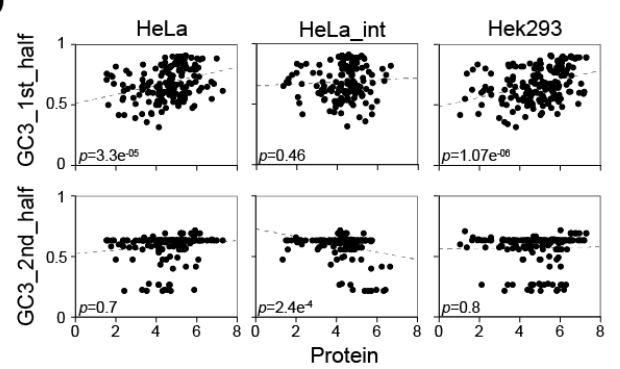

B

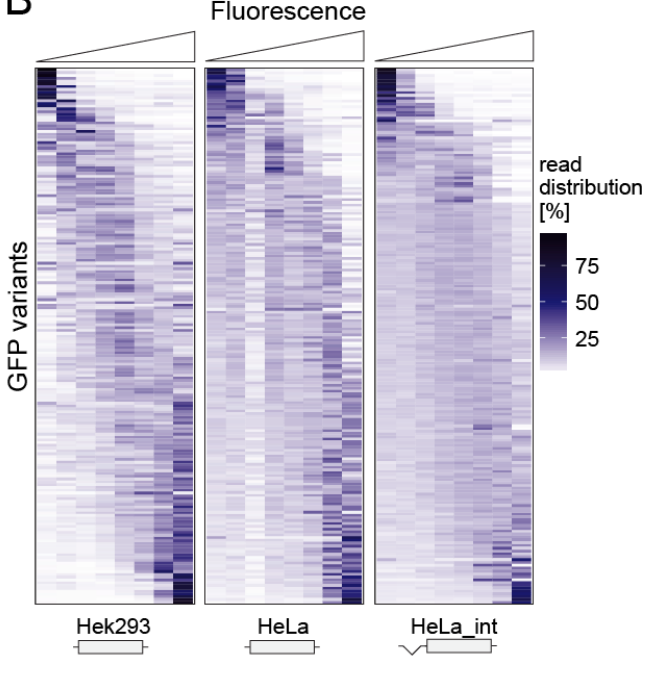

$\mathrm{E}$

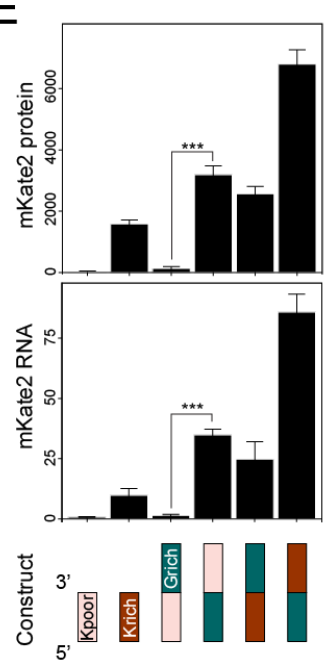

914 Figure 3. Splicing- and position-dependent effects of codon usage on 915 protein production.

916 (A) Schematic outline of Flow-Seq experimental workflow. Stable HeLa and

917 HEK293 cell pools expressing 217 GFP variants were established using a

918 multiplex Flp-In integration approach. 24h post-induction, cells are sorted by

919 FACS into 8 fluorescence bins, genomic DNA extracted followed by high-

920 throughput sequencing of the GFP locus. Individual fluorescence scores for each

921 variant are calculated from normalised read distributions. (See Methods and

922 Figure S4).

923 (B) Heatmap representation of Flow-Seq results. Rows represent normalised 924 read distributions of individual GFP variants across 8 fluorescence bins

925 (columns). The average difference between lowest and highest fluorescence bin 
926 equals about 100-fold. Data shown represents the average of 3 Flow-Seq

927 measurements for HeLa cells, the average of 3 Flow-Seq experiments for HeLa

928 with intron and 1 experiment for HEK293 cells.

929 (C) Pearson's correlation matrix of experimental measurements obtained by

930 Flow-Seq and sequence covariates. The colour of squares indicates the

931 correlation coefficient; crosses indicate non-significant correlations.

932 (D) Correlations between Flow-Seq measurements and GC3 content of $1^{\text {st }}$ (nt 1-

933 360) and $2^{\text {nd }}$ (nt 361 - 720) halves of GFP sequences.

934 (E) Protein and mRNA measurements of translational fusion constructs between

935 GC-poor (30\% GC3, Kpoor) and GC-rich (85\% GC3, Krich) variants of mKate2

936 with a GC-rich variant of GFP ( $97 \%$ GC3, Grich). Data represents the mean of 3

937 replicates + SEM (see also Figure S5).

938 
A

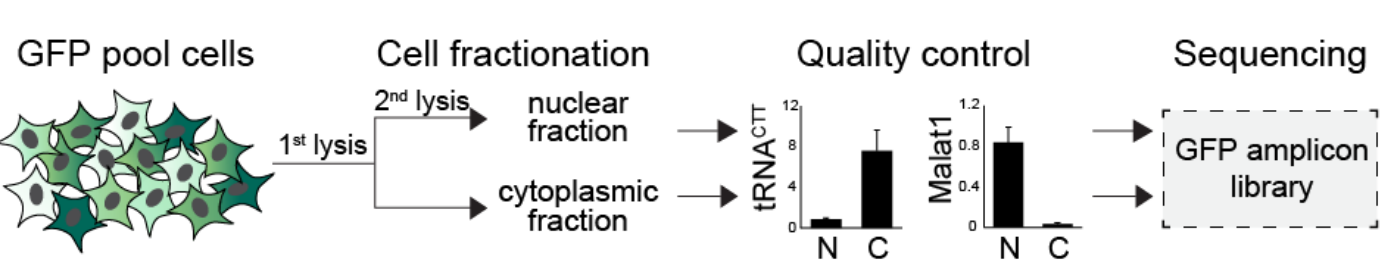

B

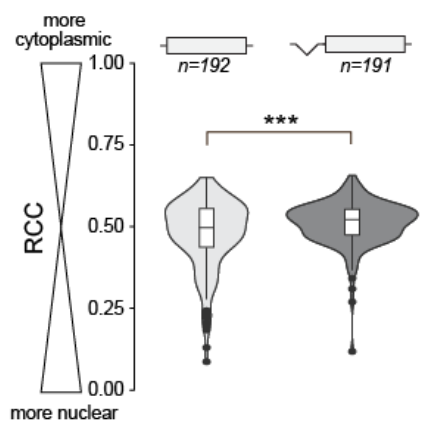

D

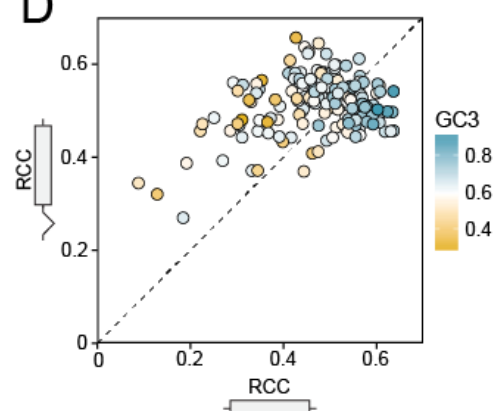

C

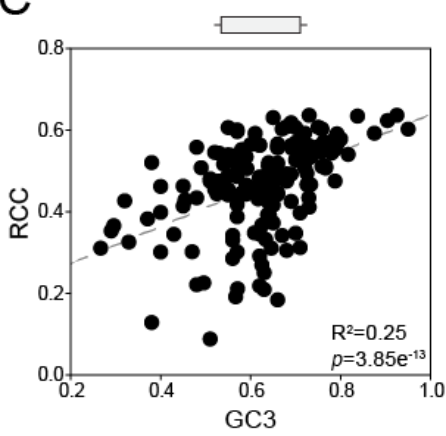

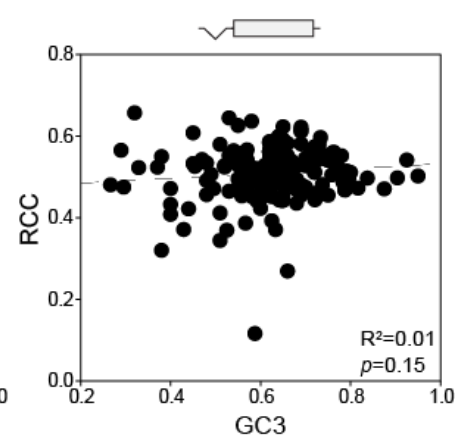

939

940

Figure 4. High GC content increases cytoplasmic localisation of mRNA.

942 (A) Stable HeLa pools expressing 217 GFP variants +/- intron were fractionated

943 into nuclear and cytoplasmic portions before RNA extraction. Specific markers of

944 subcellular compartments were quantified by qRT-PCR before amplicon-library 945 preparation (see also Methods).

946 (B) Relative cytoplasmic concentration (RCC) of unspliced and spliced GFP

947 variants. Data represents the mean of 2 replicates. ${ }^{* * *} \mathrm{p}=2 \times 10^{-6}$.

948 (C) Correlation between GC3 content and RCC for unspliced and spliced GFP

949 RNA. Data points represent the means of 2 replicates.

950 (D) Correlation between RCC scores of unspliced and spliced GFP $\left(\mathrm{R}^{2}=0.1\right.$,

$\left.951 \mathrm{p}=2.6 \times 10^{-5}\right)$. 
A

C
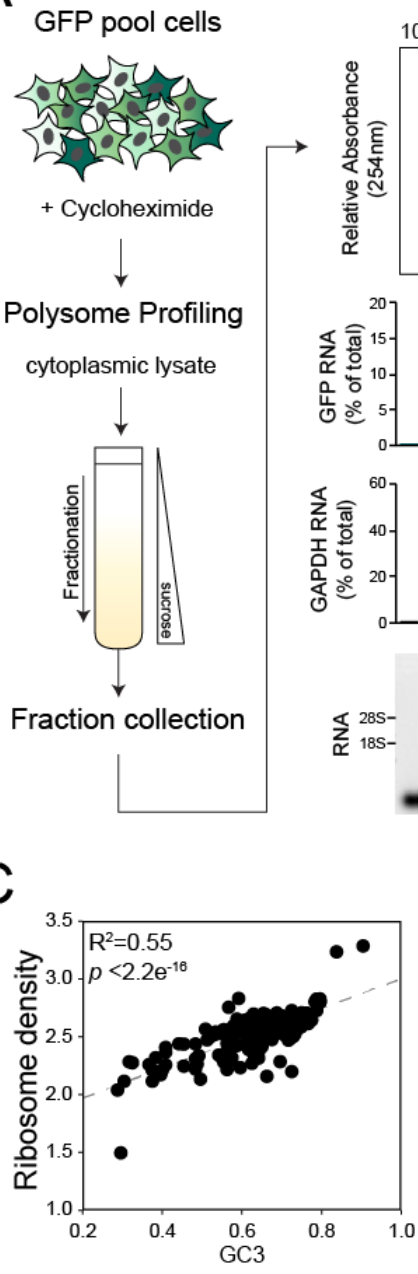
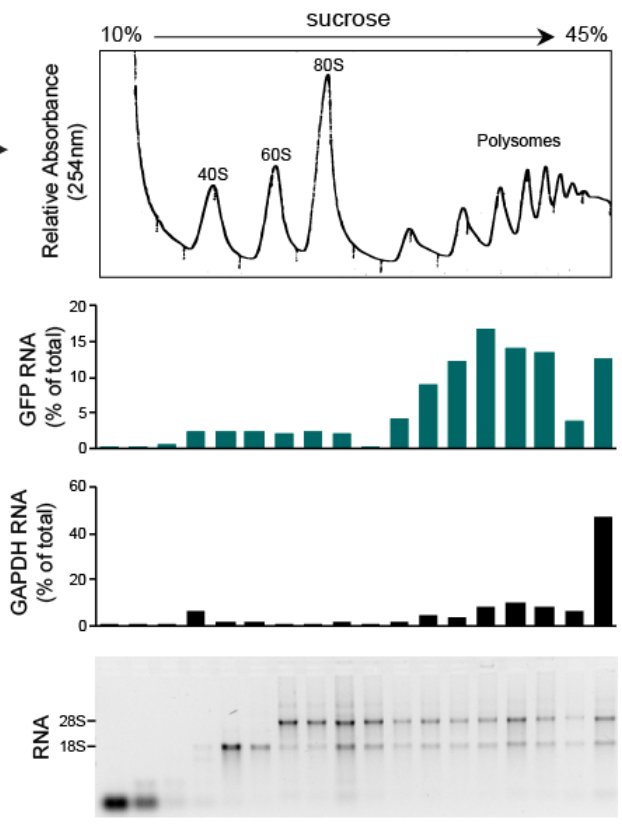

Fractions

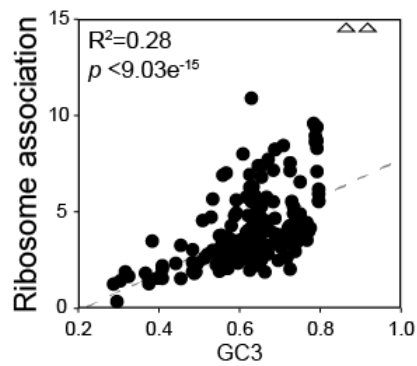

B

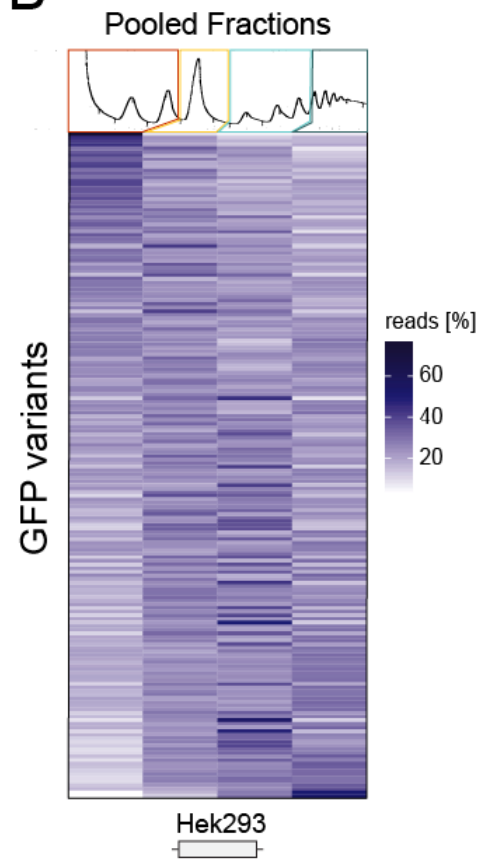

Figure 5. High GC content leads to increased ribosome association.

(A) (Left) A stable pool of HEK293 cells expressing 217 unspliced GFP variants was subjected to polysome profiling using sucrose gradient centrifugation. (Right, from top to bottom) UV absorbance profile, GFP mRNA abundance, GAPDH mRNA abundance, ethidium bromide staining of gradient fractions. GFP and GAPDH mRNA were quantified by qRT-PCR.

(B) RNA from collected fractions was combined into 4 pools (as indicated by coloured boxes) before amplicon library preparation for high-throughput sequencing: unbound ribonucleoprotein complexes (red), monosomes (yellow), light polysomes (light green) and heavy polysomes (dark green). Resulting read distributions (in \%) for GFP variants are represented as heatmap.

(C) Correlation plot between mean ribosome density (left panel) and ribosome 
A

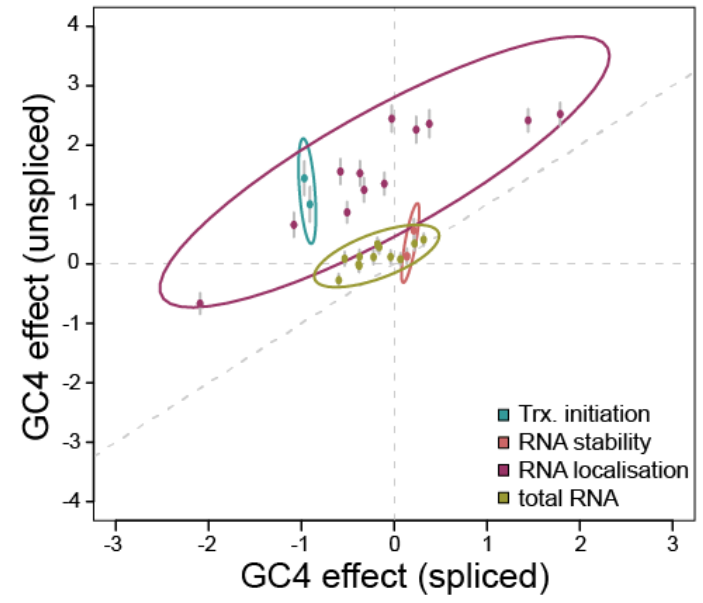

B

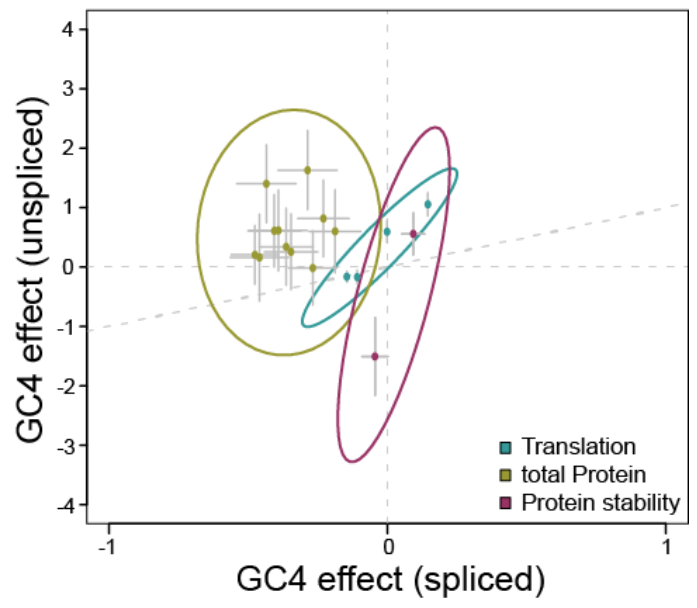

970

971 Figure 6. Splicing-dependent codon usage shapes global gene expression.

972 Plots showing the effect of GC4 content on the expression of unspliced (x-axis)

973 and spliced (y-axis) endogenous human genes, both on RNA and protein level.

974 Each point corresponds to the regression coefficient of an individual experiment

975 (cell line and/or biological replicate). Error bars indicate the standard error of

976 these regression coefficients. Surrounding ellipses indicate the 95\% confidence

977 interval for 1,000 bootstraps of underlying data (see Methods, Figure S6 and

978 Table S1). The diagonal indicates $\mathrm{x}=\mathrm{y}$. 
A

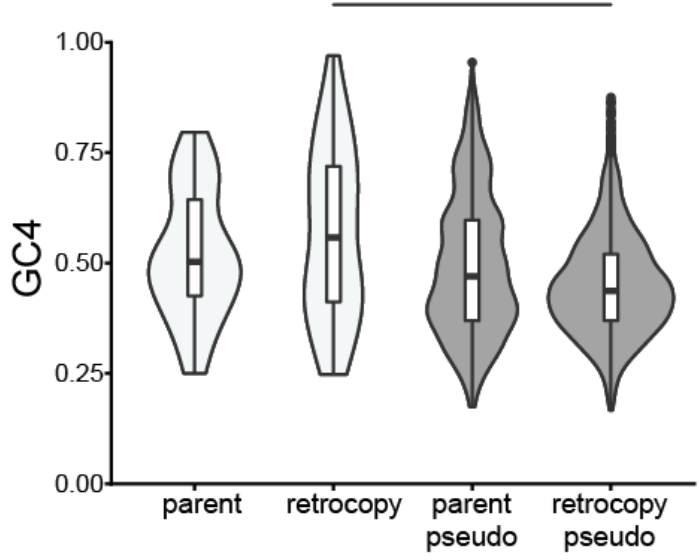

B

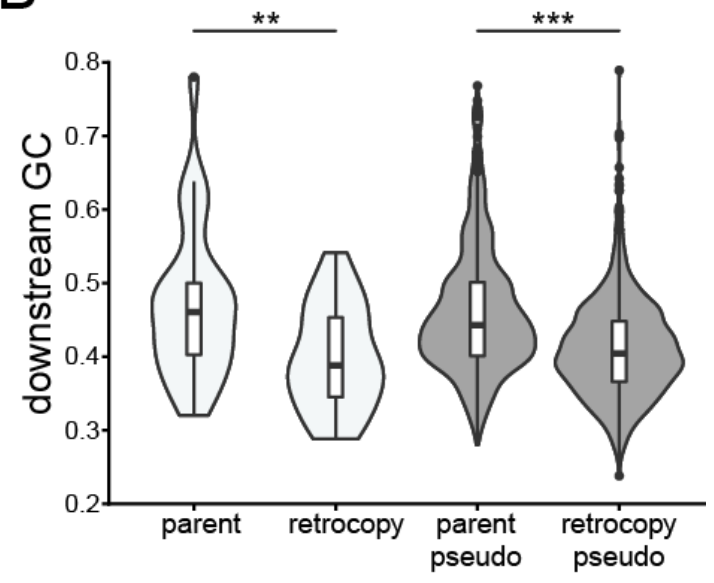

981

982

Supplementary Figure 1. GC4 variation amongst parent-retrogene pairs and their downstream sequence.

984 (A) GC4 content distribution across parent and retrogene pairs conserved 985 between human and macaque. White violins indicate pairs for which retrocopies are classed as functional ( $\mathrm{p}=0.26, \mathrm{n}=31$, two-tailed Wilcoxon signed-rank test), 987 whereas grey violins correspond to pairs in which the retrocopy is classed as 988 non-functional pseudogene ( $\mathrm{p}<2.2 \times 10^{-16}, \mathrm{n}=1562$, two-tailed Wilcoxon signed989 rank test). Note that a different retrogene dataset was used than in the main text 990 (see Methods for details). For the human-macaque set, the difference in GC4 991 between parents and functional copies is in the expected direction but not 992 significant.

993 (B) Violin plot showing GC content within a window between 2000 and 3000nt 994 downstream from the stop codons of functional (white, $p=9.27 \times 10^{-4}, \mathrm{n}=31$, two995 tailed Wilcoxon signed-rank test) and non-functional (grey, $\mathrm{p}<2.2 \times 10^{-16}, \mathrm{n}=1562$, 996 two-tailed Wilcoxon signed-rank test) parent-retrogene pairs conserved 997 between human and macaque. 
A

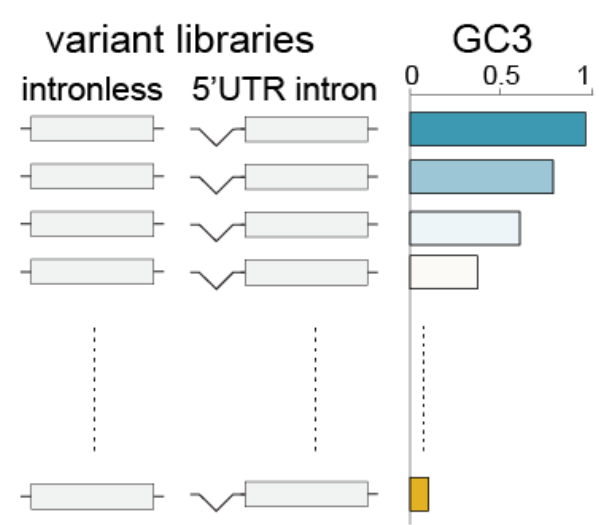

C

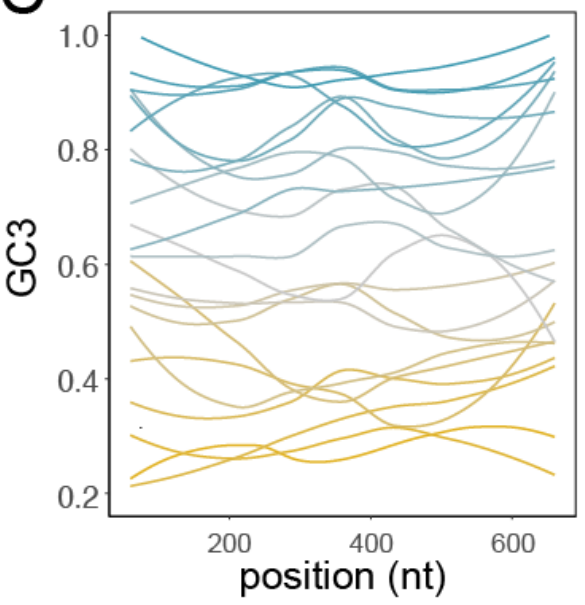

E

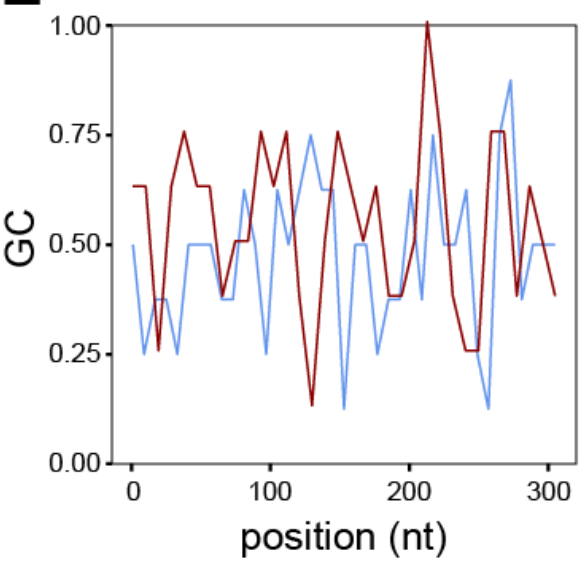

B
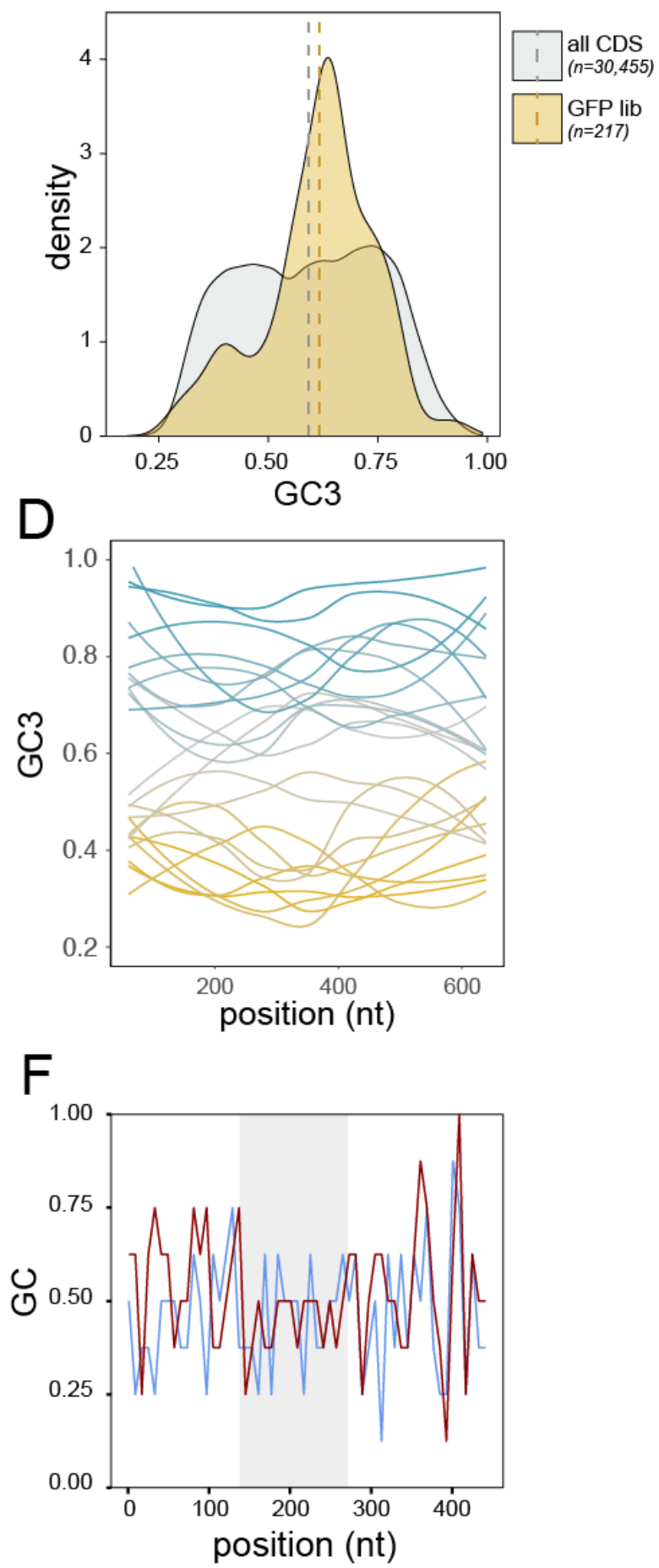

1001 Supplementary Figure 2. GC content variation amongst endogenous genes and reporter libraries.

1003 (A) Libraries of reporter genes with random synonymous codon usage were 1004 designed to cover a broad range of GC3 content variation. Variants were 1005 expressed with and without a synthetic 5'UTR intron. 
1006 (B) GC3 content distribution amongst human consensus coding sequences (CDS;

1007 grey) in comparison to the GFP variant library used in this study (GFP lib;

1008 orange). Dashed lines indicate the mean GC3 for each data set.

1009 (C-D) Loess-smoothed GC3 profiles along the 22 GFP variants (C) and 23 mKate

1010 variants (D) that were analysed by spectrofluorometry (Figure 2).

1011 (E) Sliding window analysis of GC content in 5'UTRs of intronless expression

1012 cassettes utilised in this study. Blue: pCM3 (transient transfection, no intron);

1013 red: pcDNA5/FRT/TO/DEST (stable transfection, no intron).

1014 (F) As above, intron-containing expression cassettes. Blue: pCM4 (transient 1015 transfection, with intron); red: pcDNA5/FRT/TO/DEST/INT (stable transfection, 1016 with intron). Grey shading indicates the position of the synthetic intron. 

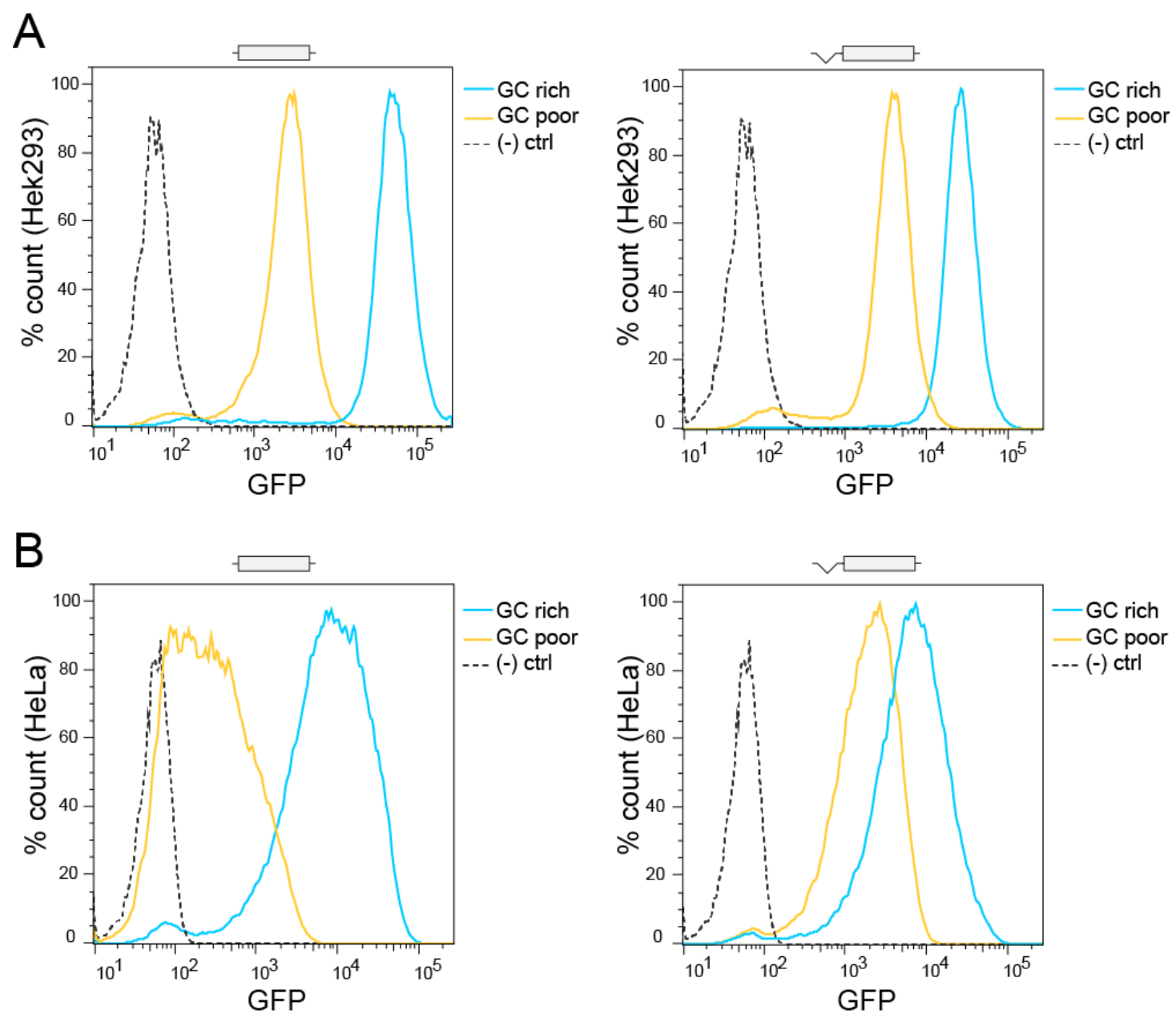

1020 Supplementary Figure 3. Effect of GC content on expression of fluorescent 1021 reporter genes in stably transfected cell lines.

1022 Flow cytometry measurements of two GFP variants in stably transfected HEK293 1023 Flp-in (A) and HeLa Flp-in (B). GC poor = 33\% GC3; GC rich = 97\% GC3; (-)ctrl = 1024 untransfected cells. Data shows representative results of at least 3 experiments. 
A

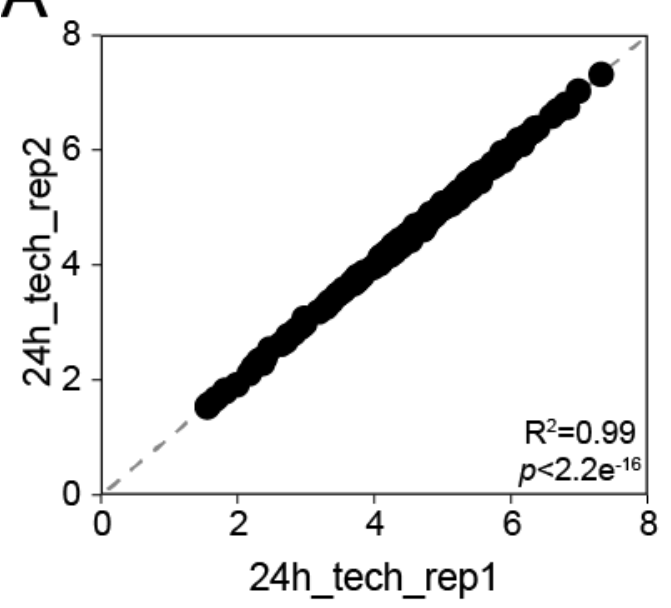

C

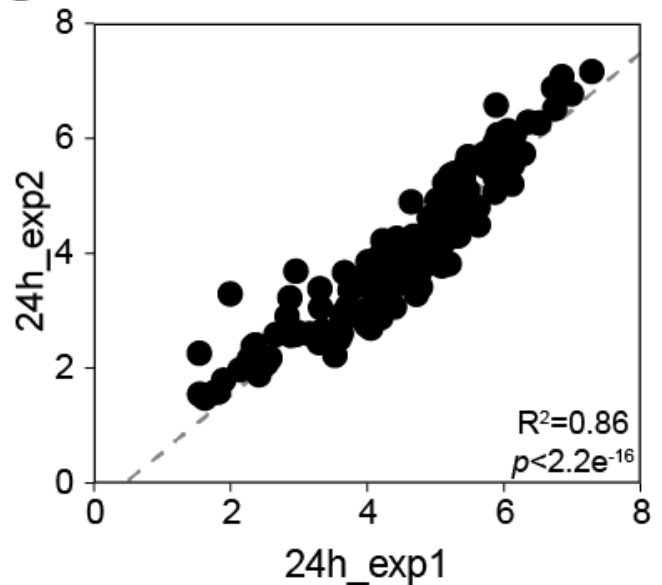

E

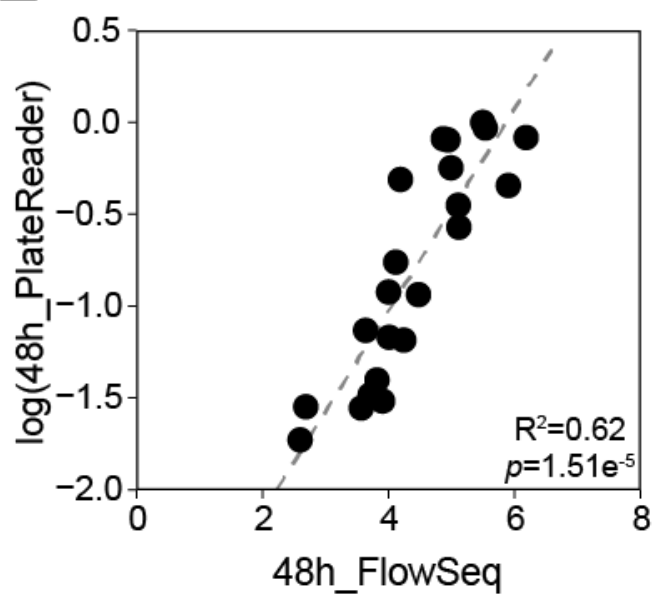

$B$

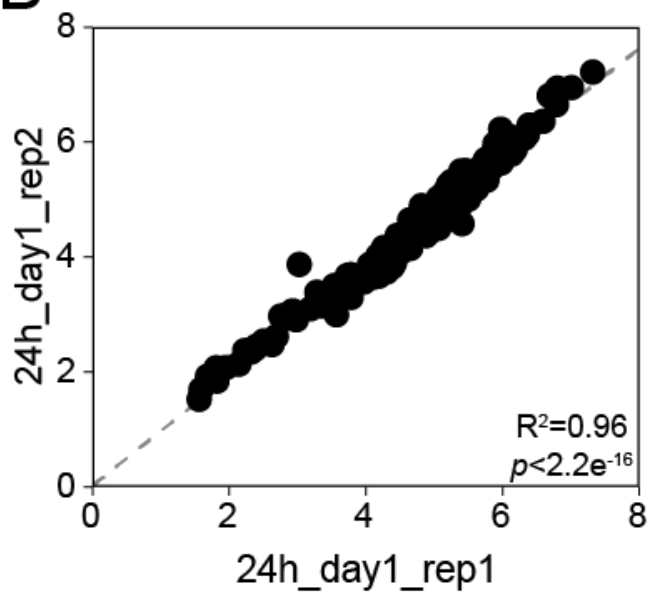

D

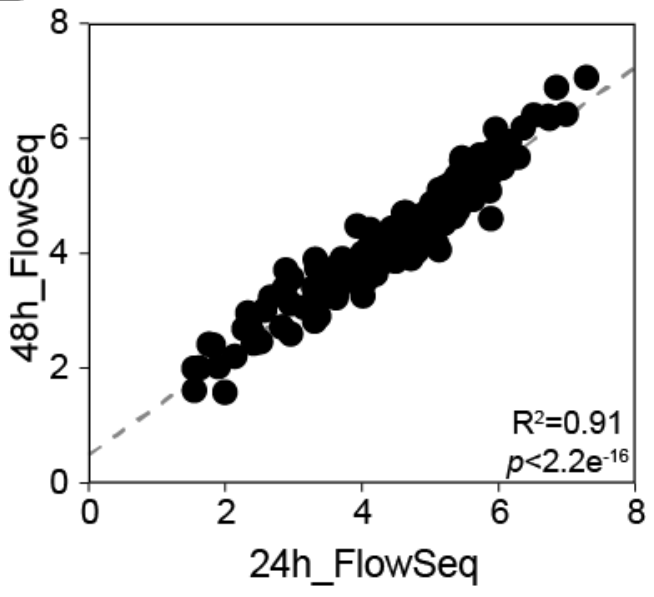

1028 Supplementary Figure 4. Reproducibility of Flow-seq experiments in HeLa 1029 cells (unspliced GFP variants).

1030 (A) Re-sequencing of the same amplicon-library.

1031 (B-C) Replicate Flow-seq experiments performed on the same day (B) or 1032 different days (C). 

available under aCC-BY 4.0 International license.

1033 (D) Flow-Seq experiments performed on the same pool of cells, $24 \mathrm{~h}$ and $48 \mathrm{~h}$ 1034 after the induction of GFP expression.

1035 (E) Correlation between fluorescence measurements of 22 GFP variants obtained 1036 spectrofluorometry of transiently transfected HeLa cells and by Flow-Seq of 1037 HeLa GFP pool cell line.

1038 
A

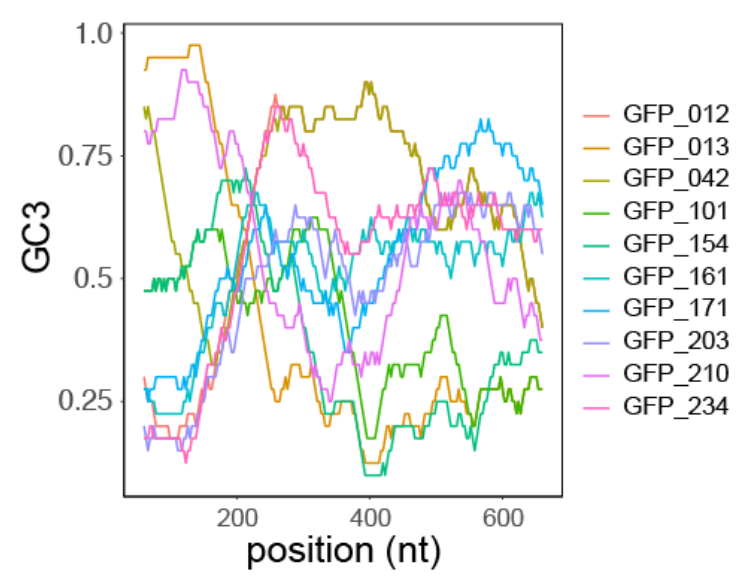

C
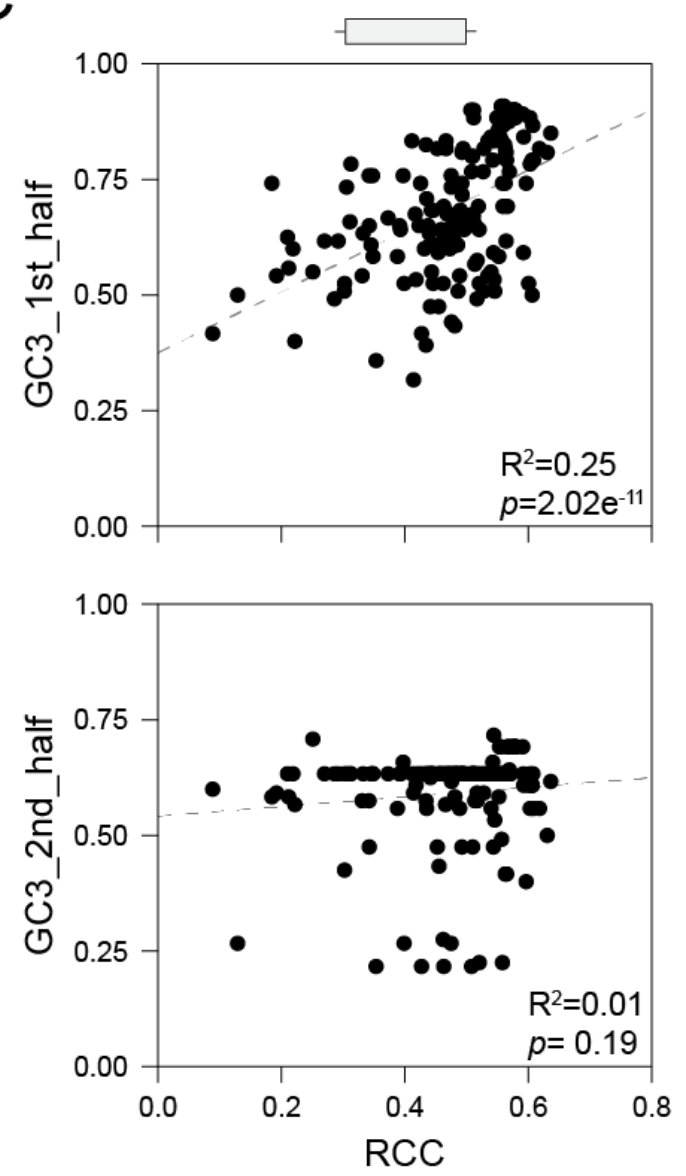

B
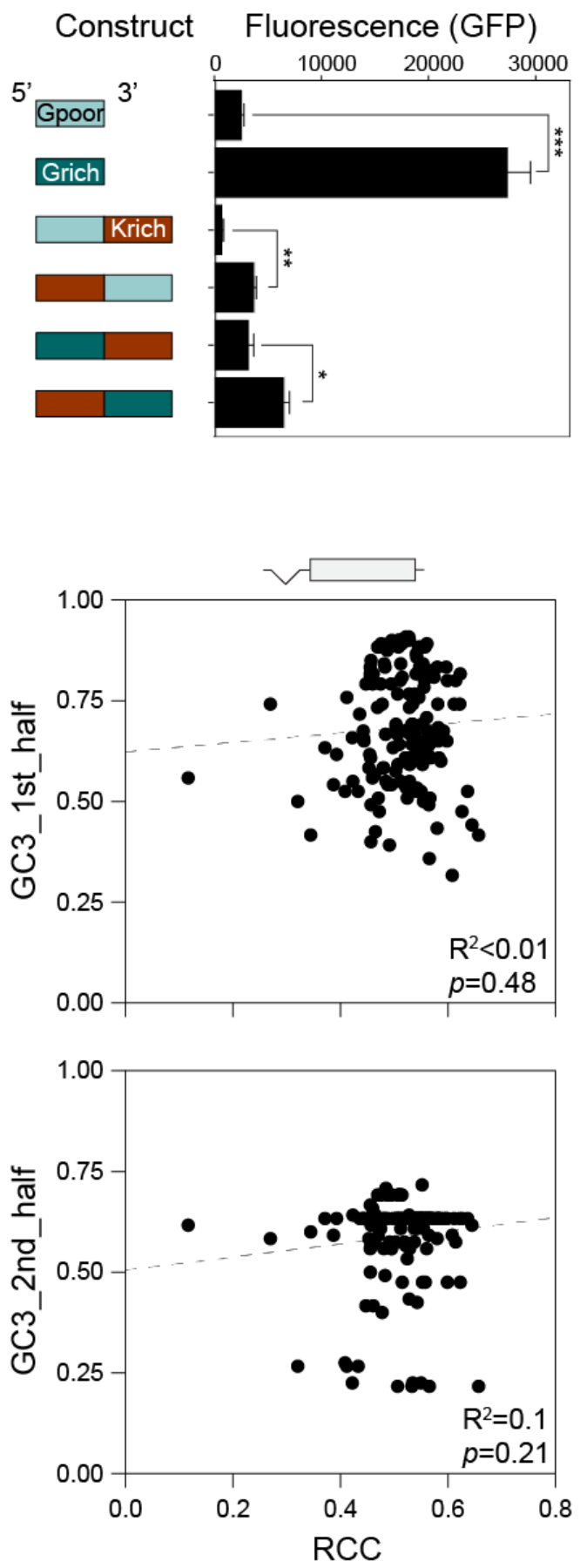

1041 Supplementary Figure 5. Position-specific effects of GC content on 1042 expression.

1043 (A) Sliding window analysis of GC3 content in selected GFP variants used in the 1044 pooled amplicon sequencing experiments.

1045 (B) Protein measurements of translational fusion constructs between GC-poor 1046 (33\% GC3, Gpoor) and GC-rich (97\% GC3, Grich) variants of GFP with a GC-rich 
1047 variant of mKate2 (85\% GC3, Krich), upon transient transfection into HeLa cells.

1048 Data represent the mean of 3 replicates + SEM.

1049 (C) Correlations between the GC3 content in the 1st (nt 1-360) and 2nd (nt 361-

1050 720) halves of GFP variants and their relative cytoplasmic mRNA concentrations. 

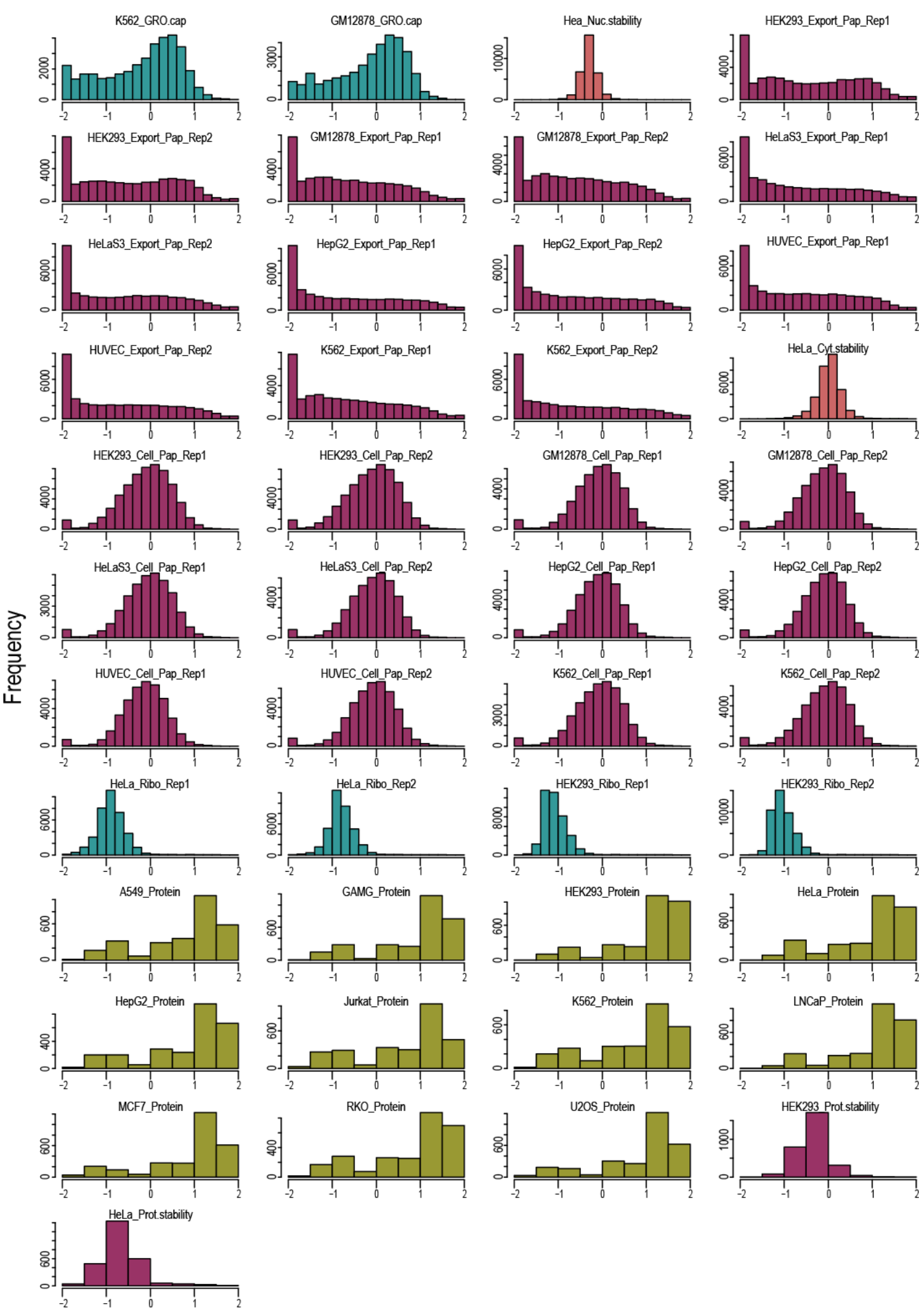

\section{Supplementary Figure 6. Distribution of RNA and protein expression data}

\section{used in regression modelling.}

Human RNA and protein expression data were extracted from various databases, filtered and normalized as described in Supplementary Table 1 and in the 
bioRxiv preprint doi: https://doi.org/10.1101/527440; this version posted January 22, 2019 . The copyright holder for this preprint (which was not certified by peer review) is the author/funder, who has granted bioRxiv a license to display the preprint in perpetuity. It is made available under aCC-BY 4.0 International license.

1058 Methods section. The histograms show the distributions of preprocessed

1059 expression measurements.

1060 


\section{Supplementary Table 1. Sources of human gene expression data.}

1062 The cellular process to be quantified is indicated above the table, and the

1063 experimental techniques and data sources are indicated below. Each dot

1064 indicates an experimental replicate measurement.

1065

\begin{tabular}{|c|c|c|c|c|c|c|c|c|}
\hline K562 & $\bullet$ & & & $\bullet \bullet$ & $\bullet \bullet$ & & $\bullet$ & \\
\hline Gm12878 & $\bullet$ & & & $\bullet \bullet$ & $\bullet \bullet$ & & & \\
\hline HeLa & & $\bullet$ & $\bullet$ & $\bullet \bullet$ & $\bullet \bullet$ & $\bullet \bullet$ & $\bullet$ & $\bullet$ \\
\hline Hek293 & & & & $\bullet \bullet$ & $\bullet \bullet$ & $\bullet \bullet$ & $\bullet$ & $\bullet$ \\
\hline Huvec & & & & $\bullet \bullet$ & $\bullet \bullet$ & & & \\
\hline HepG2 & & & & $\bullet \bullet$ & $\bullet \bullet$ & & $\bullet$ & \\
\hline A549 & & & & & & & $\bullet$ & \\
\hline GAMG & & & & & & & $\bullet$ & \\
\hline Jurkat & & & & & & & $\bullet$ & \\
\hline LnCap & & & & & & & $\bullet$ & \\
\hline MCF7 & & & & & & & $\bullet$ & \\
\hline RKO & & & & & & & $\bullet$ & \\
\hline U2OS & & & & & & & $\bullet$ & \\
\hline data type & GRO-cap & $\begin{array}{l}\text { CAGE-seq: } \\
\text { Mtr4 KD/ } \\
\text { EGFP KD }\end{array}$ & $\begin{array}{l}\text { CAGE-seq: } \\
\text { Rrp40 KD/ } \\
\text { Mtr4 KD }\end{array}$ & RNA-seq & RNA-seq & Ribo-seq & Mass-spec & $\begin{array}{l}\text { Mass- } \\
\text { spec/Ribo- } \\
\text { seq }\end{array}$ \\
\hline $\begin{array}{c}\text { data } \\
\text { source }\end{array}$ & ENCODE & $\begin{array}{l}\text { Andersson } \\
\text { et al., 2014 }\end{array}$ & $\begin{array}{l}\text { Andersson } \\
\text { et al., } 2014\end{array}$ & $\begin{array}{l}\text { Hek293: } \\
\text { this study; } \\
\text { all others: } \\
\text { ENCODE }\end{array}$ & $\begin{array}{l}\text { Hek293: } \\
\text { this study; } \\
\text { all others: } \\
\text { ENCODE }\end{array}$ & ENCODE & $\begin{array}{l}\text { Geiger et } \\
\text { al., } 2012\end{array}$ & $\begin{array}{l}\text { Geiger et } \\
\text { al., 2012; } \\
\text { ENCODE }\end{array}$ \\
\hline
\end{tabular}




\section{Supplementary Table 2. List of primers used.}

1069

\begin{tabular}{|c|c|}
\hline $\begin{array}{l}\text { MiSeq library + } \\
\text { sequencing }\end{array}$ & $5^{\prime} \rightarrow 3^{\prime}$ \\
\hline PE_PCR_left & $\begin{array}{l}\text { AATGATACGGCGACCACCGAGATCTACACGCTGGCACGCGTAAGAAGGAGATATAACCAT } \\
\text { G }\end{array}$ \\
\hline $\begin{array}{l}\text { S_index1_right_P } \\
\text { EPCR }\end{array}$ & $\begin{array}{l}\text { CAAGCAGAAGACGGCATACGAGATCGTGATGTGACTGGAGTTCAGACGTGTGCTCTTCC } \\
\text { GATCTATGTGCAGGGCCGCGAATTC }\end{array}$ \\
\hline $\begin{array}{l}\text { S_index2_right_P } \\
\text { EPCR }\end{array}$ & $\begin{array}{l}\text { CAAGCAGAAGACGGCATACGAGATACATCGGTGACTGGAGTTCAGACGTGTGCTCTTCC } \\
\text { GATCTATGTGCAGGGCCGCGAATTC }\end{array}$ \\
\hline $\begin{array}{l}\text { S_index3_right_P } \\
\text { EPCR }\end{array}$ & $\begin{array}{l}\text { CAAGCAGAAGACGGCATACGAGATGCCTAAGTGACTGGAGTTCAGACGTGTGCTCTTCC } \\
\text { GATCTATGTGCAGGGCCGCGAATTC }\end{array}$ \\
\hline $\begin{array}{l}\text { S_index4_right_P } \\
\text { EPCR }\end{array}$ & $\begin{array}{l}\text { CAAGCAGAAGACGGCATACGAGATTGGTCAGTGACTGGAGTTCAGACGTGTGCTCTTCC } \\
\text { GATCTATGTGCAGGGCCGCGAATTC }\end{array}$ \\
\hline $\begin{array}{l}\text { S_index5_right_P } \\
\text { EPCR }\end{array}$ & $\begin{array}{l}\text { CAAGCAGAAGACGGCATACGAGATCACTGTGTGACTGGAGTTCAGACGTGTGCTCTTCC } \\
\text { GATCTATGTGCAGGGCCGCGAATTC }\end{array}$ \\
\hline $\begin{array}{l}\text { S_index6_right_P } \\
\text { EPCR }\end{array}$ & $\begin{array}{l}\text { CAAGCAGAAGACGGCATACGAGATATTGGCGTGACTGGAGTTCAGACGTGTGCTCTTCC } \\
\text { GATCTATGTGCAGGGCCGCGAATTC }\end{array}$ \\
\hline $\begin{array}{l}\text { S_index7_right_P } \\
\text { EPCR }\end{array}$ & $\begin{array}{l}\text { CAAGCAGAAGACGGCATACGAGATGATCTGGTGACTGGAGTTCAGACGTGTGCTCTTCC } \\
\text { GATCTATGTGCAGGGCCGCGAATTC }\end{array}$ \\
\hline $\begin{array}{l}\text { S_index8_right_P } \\
\text { EPCR }\end{array}$ & $\begin{array}{l}\text { CAAGCAGAAGACGGCATACGAGATTCAAGTGTGACTGGAGTTCAGACGTGTGCTCTTCCG } \\
\text { ATCTATGTGCAGGGCCGCGAATTC }\end{array}$ \\
\hline $\begin{array}{l}\text { Read1_seq_prim } \\
\text { er_GFP }\end{array}$ & GCTGGCACGCGTAAGAAGGAGATATAACCATG \\
\hline \multicolumn{2}{|l|}{ cloning primers } \\
\hline $\begin{array}{l}\text { pCl_del_int_F } \\
\text { (phospho) }\end{array}$ & GTGTCCACTCCCAGTTCAAT \\
\hline $\begin{array}{l}\text { pCl_del_int_R } \\
\text { (phospho) }\end{array}$ & CTGCCCAGTGCCTCACGACC \\
\hline mkate2_gibs_F & GATCCGCGTATGGTGGCCTTAAGATACATTGATGAG \\
\hline mkate2_gibs_R & TGTAAGCGGATGCCGCACATGTTCTTTCCTGCG \\
\hline pCl_gib_F & CGGCATCCGCTTACAGACAA \\
\hline pCl_gib_R & CACCATACGCGGATCCTTATC \\
\hline \multicolumn{2}{|l|}{ qPCR primers } \\
\hline pcDNA5-UTR_F & GTTGCCAGCCATCTGTTGTT \\
\hline pcDNA5-UTR_R & CTCAGACAATGCGATGCAATTTCC \\
\hline pCl-UTR_F & СTTCCСTTTAGTGAGGGTTAATG \\
\hline pCl-UTR_R & GTTTATTGCAGCTTATAATGGTTAC \\
\hline pCl-mRNA_F & GCTAACGCAGTCAGTGCTTC \\
\hline pCl-mRNA_R & ACACCCAGTGCCTCACGAC \\
\hline pCl-premRNA_F & GAGGCACTGGGCAGGTAAGTATC \\
\hline pCl-premRNA_R & GTGGATGTCAGTAAGACCAATAGGTG \\
\hline Gapdh_F & GGAGTCAACGGATTTGG \\
\hline Gapdh_R & GTAGTTGAGGTCAATGAAGGG \\
\hline Neo_F & CCCGTGATATTGCTGAAGAG \\
\hline Neo_R & CGTCAAGAAGGCGATAGAAG \\
\hline LysCTT_F & TCAGTCGGTAGAGCATGAGAC \\
\hline LysCTT_R & CAACGTGGGGCTCGAACC \\
\hline Malat1_F & CAGACССТTCAССССТСАС \\
\hline Malat1_R & TTATGGATCATGCCCACAAG \\
\hline
\end{tabular}




\section{Reference list}

Andersson, R., Refsing Andersen, P., Valen, E., Core, L.J., Bornholdt, J., Boyd, M., Heick Jensen, T., and Sandelin, A. (2014). Nuclear stability and transcriptional directionality separate functionally distinct RNA species. Nature communications 5,5336 .

Arango, D., Sturgill, D., Alhusaini, N., Dillman, A.A., Sweet, T.J., Hanson, G., Hosogane, M., Sinclair, W.R., Nanan, K.K., Mandler, M.D., et al. (2018). Acetylation of Cytidine in mRNA Promotes Translation Efficiency. Cell 175, 1872-1886 e1824.

Arhondakis, S., Auletta, F., and Bernardi, G. (2011). Isochores and the regulation of gene expression in the human genome. Genome Biol Evol 3, 1080-1089.

Bauer, A.P., Leikam, D., Krinner, S., Notka, F., Ludwig, C., Langst, G., and Wagner, R. (2010). The impact of intragenic CpG content on gene expression. Nucleic Acids Res 38, 3891-3908.

Bazzini, A.A., Del Viso, F., Moreno-Mateos, M.A., Johnstone, T.G., Vejnar, C.E., Qin, Y., Yao, J., Khokha, M.K., and Giraldez, A.J. (2016). Codon identity regulates mRNA stability and translation efficiency during the maternal-to-zygotic transition. EMBO J 35, 2087-2103.

Bentele, K., Saffert, P., Rauscher, R., Ignatova, Z., and Bluthgen, N. (2013). Efficient translation initiation dictates codon usage at gene start. Mol Syst Biol 9, 675.

Bernardi, G. (1993). The vertebrate genome: isochores and evolution. Mol Biol Evol 10, 186-204.

Burow, D.A., Martin, S., Quail, J.F., Alhusaini, N., Coller, J., and Cleary, M.D. (2018). Attenuated Codon Optimality Contributes to Neural-Specific mRNA Decay in Drosophila. Cell reports 24, 1704-1712.

Carels, N., and Bernardi, G. (2000). Two classes of genes in plants. Genetics 154, 1819-1825.

Dittmar, K.A., Goodenbour, J.M., and Pan, T. (2006). Tissue-specific differences in human transfer RNA expression. PLoS Genet 2, e221.

Dominissini, D., Moshitch-Moshkovitz, S., Schwartz, S., Salmon-Divon, M., Ungar, L., Osenberg, S., Cesarkas, K., Jacob-Hirsch, J., Amariglio, N., Kupiec, M., et al. (2012). Topology of the human and mouse m6A RNA methylomes revealed by m6A-seq. Nature 485, 201-206.

dos Reis, M., Savva, R., and Wernisch, L. (2004). Solving the riddle of codon usage preferences: a test for translational selection. Nucleic Acids Res 32, 5036-5044.

Duan, J., Shi, J., Ge, X., Dolken, L., Moy, W., He, D., Shi, S., Sanders, A.R., Ross, J., and Gejman, P.V. (2013). Genome-wide survey of interindividual differences of RNA stability in human lymphoblastoid cell lines. Scientific reports 3, 1318.

Duret, L., and Galtier, N. (2009). Biased gene conversion and the evolution of mammalian genomic landscapes. Annu Rev Genomics Hum Genet 10, 285-311. 
Eyre-Walker, A.C. (1991). An analysis of codon usage in mammals: selection or mutation bias? J Mol Evol 33, 442-449.

Fath, S., Bauer, A.P., Liss, M., Spriestersbach, A., Maertens, B., Hahn, P., Ludwig, C., Schafer, F., Graf, M., and Wagner, R. (2011). Multiparameter RNA and codon optimization: a standardized tool to assess and enhance autologous mammalian gene expression. PLoS One 6, e17596.

Gagnon, K.T., Li, L., Janowski, B.A., and Corey, D.R. (2014). Analysis of nuclear RNA interference in human cells by subcellular fractionation and Argonaute loading. Nat Protoc 9, 2045-2060.

Galtier, N., Roux, C., Rousselle, M., Romiguier, J., Figuet, E., Glemin, S., Bierne, N., and Duret, L. (2018). Codon Usage Bias in Animals: Disentangling the Effects of Natural Selection, Effective Population Size, and GC-Biased Gene Conversion. Mol Biol Evol 35, 1092-1103.

Geiger, T., Wehner, A., Schaab, C., Cox, J., and Mann, M. (2012). Comparative proteomic analysis of eleven common cell lines reveals ubiquitous but varying expression of most proteins. Mol Cell Proteomics 11, M111 014050.

Gingold, H., Tehler, D., Christoffersen, N.R., Nielsen, M.M., Asmar, F., Kooistra, S.M., Christophersen, N.S., Christensen, L.L., Borre, M., Sorensen, K.D., et al. (2014). A dual program for translation regulation in cellular proliferation and differentiation. Cell 158, 1281-1292.

Goodman, D.B., Church, G.M., and Kosuri, S. (2013). Causes and effects of Nterminal codon bias in bacterial genes. Science 342, 475-479.

Gu, W., Zhou, T., and Wilke, C.O. (2010). A universal trend of reduced mRNA stability near the translation-initiation site in prokaryotes and eukaryotes. PLoS Comput Biol 6, e1000664.

Higgs, D.R., Goodbourn, S.E., Lamb, J., Clegg, J.B., Weatherall, D.J., and Proudfoot, N.J. (1983). Alpha-thalassaemia caused by a polyadenylation signal mutation. Nature 306, 398-400.

Kosovac, D., Wild, J., Ludwig, C., Meissner, S., Bauer, A.P., and Wagner, R. (2011). Minimal doses of a sequence-optimized transgene mediate high-level and longterm EPO expression in vivo: challenging CpG-free gene design. Gene Ther 18, 189-198.

Kosuri, S., Goodman, D.B., Cambray, G., Mutalik, V.K., Gao, Y., Arkin, A.P., Endy, D., and Church, G.M. (2013). Composability of regulatory sequences controlling transcription and translation in Escherichia coli. Proc Natl Acad Sci U S A 110, 14024-14029.

Kotsopoulou, E., Kim, V.N., Kingsman, A.J., Kingsman, S.M., and Mitrophanous, K.A. (2000). A Rev-independent human immunodeficiency virus type 1 (HIV-1)based vector that exploits a codon-optimized HIV-1 gag-pol gene. J Virol 74, 4839-4852.

Kudla, G., Lipinski, L., Caffin, F., Helwak, A., and Zylicz, M. (2006). High guanine and cytosine content increases mRNA levels in mammalian cells. PLoS Biol 4, e180. 
Kudla, G., Murray, A.W., Tollervey, D., and Plotkin, J.B. (2009). Coding-sequence determinants of gene expression in Escherichia coli. Science 324, 255-258.

Kwek, K.Y., Murphy, S., Furger, A., Thomas, B., O'Gorman, W., Kimura, H., Proudfoot, N.J., and Akoulitchev, A. (2002). U1 snRNA associates with TFIIH and regulates transcriptional initiation. Nat Struct Biol 9, 800-805.

Lander, E.S., Linton, L.M., Birren, B., Nusbaum, C., Zody, M.C., Baldwin, J., Devon, K., Dewar, K., Doyle, M., FitzHugh, W., et al. (2001). Initial sequencing and analysis of the human genome. Nature 409, 860-921.

Lercher, M.J., Urrutia, A.O., Pavlicek, A., and Hurst, L.D. (2003). A unification of mosaic structures in the human genome. Hum Mol Genet 12, 2411-2415.

Li, W. (2011). On parameters of the human genome. J Theor Biol 288, 92-104.

Livak, K.J., and Schmittgen, T.D. (2001). Analysis of relative gene expression data using real-time quantitative PCR and the 2(-Delta Delta C(T)) Method. Methods $25,402-408$.

Lubelsky, Y., and Ulitsky, I. (2018). Sequences enriched in Alu repeats drive nuclear localization of long RNAs in human cells. Nature 555, 107-111.

Mishima, Y., and Tomari, Y. (2016). Codon Usage and 3' UTR Length Determine Maternal mRNA Stability in Zebrafish. Mol Cell 61, 874-885.

Mittal, P., Brindle, J., Stephen, J., Plotkin, J.B., and Kudla, G. (2018). Codon usage influences fitness through RNA toxicity. Proc Natl Acad Sci U S A 115, 8639-8644.

Muller-McNicoll, M., Botti, V., de Jesus Domingues, A.M., Brandl, H., Schwich, O.D., Steiner, M.C., Curk, T., Poser, I., Zarnack, K., and Neugebauer, K.M. (2016). SR proteins are NXF1 adaptors that link alternative RNA processing to mRNA export. Genes Dev 30, 553-566.

Nott, A., Le Hir, H., and Moore, M.J. (2004). Splicing enhances translation in mammalian cells: an additional function of the exon junction complex. Genes Dev $18,210-222$.

Nott, A., Meislin, S.H., and Moore, M.J. (2003). A quantitative analysis of intron effects on mammalian gene expression. RNA 9, 607-617.

Palazzo, A.F., and Akef, A. (2012). Nuclear export as a key arbiter of "mRNA identity" in eukaryotes. Biochim Biophys Acta 1819, 566-577.

Palazzo, A.F., Springer, M., Shibata, Y., Lee, C.S., Dias, A.P., and Rapoport, T.A. (2007). The signal sequence coding region promotes nuclear export of mRNA. PLoS Biol 5, e322.

Parmley, J.L., Urrutia, A.O., Potrzebowski, L., Kaessmann, H., and Hurst, L.D. (2007). Splicing and the evolution of proteins in mammals. PLoS biology 5, e14.

Plotkin, J.B., and Kudla, G. (2011). Synonymous but not the same: the causes and consequences of codon bias. Nat Rev Genet 12, 32-42.

Plotkin, J.B., Robins, H., and Levine, A.J. (2004). Tissue-specific codon usage and the expression of human genes. Proc Natl Acad Sci U S A 101, 12588-12591. 
Presnyak, V., Alhusaini, N., Chen, Y.H., Martin, S., Morris, N., Kline, N., Olson, S., Weinberg, D., Baker, K.E., Graveley, B.R., et al. (2015). Codon optimality is a major determinant of mRNA stability. Cell 160,1111-1124.

R Development Core Team (2005). R: A language and environment for statistical computing (Vienna, Austria: R Foundation for Statistical Computing).

Radhakrishnan, A., Chen, Y.H., Martin, S., Alhusaini, N., Green, R., and Coller, J. (2016). The DEAD-Box Protein Dhh1p Couples mRNA Decay and Translation by Monitoring Codon Optimality. Cell 167, 122-132 e129.

Ressayre, A., Glemin, S., Montalent, P., Serre-Giardi, L., Dillmann, C., and Joets, J. (2015). Introns Structure Patterns of Variation in Nucleotide Composition in Arabidopsis thaliana and Rice Protein-Coding Genes. Genome Biol Evol 7, 29132928.

Rosikiewicz, W., Kabza, M., Kosinski, J.G., Ciomborowska-Basheer, J., Kubiak, M.R., and Makalowska, I. (2017). RetrogeneDB-a database of plant and animal retrocopies. Database (Oxford) 2017.

Rudolph, K.L., Schmitt, B.M., Villar, D., White, R.J., Marioni, J.C., Kutter, C., and Odom, D.T. (2016). Codon-Driven Translational Efficiency Is Stable across Diverse Mammalian Cell States. PLoS Genet 12, e1006024.

Savisaar, R., and Hurst, L.D. (2016). Purifying Selection on Exonic Splice Enhancers in Intronless Genes. Mol Biol Evol 33, 1396-1418.

Semon, M., Mouchiroud, D., and Duret, L. (2005). Relationship between gene expression and GC-content in mammals: statistical significance and biological relevance. Hum Mol Genet 14, 421-427.

Shah, P., Ding, Y., Niemczyk, M., Kudla, G., and Plotkin, J.B. (2013). Rate-limiting steps in yeast protein translation. Cell 153, 1589-1601.

Sharp, P.M., and Li, W.H. (1987a). The codon Adaptation Index--a measure of directional synonymous codon usage bias, and its potential applications. Nucleic Acids Res 15, 1281-1295.

Sharp, P.M., and Li, W.H. (1987b). The rate of synonymous substitution in enterobacterial genes is inversely related to codon usage bias. Mol Biol Evol 4, 222-230.

Takata, M.A., Goncalves-Carneiro, D., Zang, T.M., Soll, S.J., York, A., Blanco-Melo, D., and Bieniasz, P.D. (2017). CG dinucleotide suppression enables antiviral defence targeting non-self RNA. Nature 550, 124-127.

Tuller, T., Carmi, A., Vestsigian, K., Navon, S., Dorfan, Y., Zaborske, J., Pan, T., Dahan, O., Furman, I., and Pilpel, Y. (2010). An evolutionarily conserved mechanism for controlling the efficiency of protein translation. Cell 141, 344354.

Vinogradov, A.E. (2003). Isochores and tissue-specificity. Nucleic Acids Res 31, 5212-5220.

Wang, Y., Zhu, W., and Levy, D.E. (2006). Nuclear and cytoplasmic mRNA quantification by SYBR green based real-time RT-PCR. Methods 39, 356-362. 
Webster, M.W., Chen, Y.H., Stowell, J.A.W., Alhusaini, N., Sweet, T., Graveley, B.R., Coller, J., and Passmore, L.A. (2018). mRNA Deadenylation Is Coupled to Translation Rates by the Differential Activities of Ccr4-Not Nucleases. Mol Cell 70, 1089-1100 e1088.

Zaghlool, A., Ameur, A., Nyberg, L., Halvardson, J., Grabherr, M., Cavelier, L., and Feuk, L. (2013). Efficient cellular fractionation improves RNA sequencing analysis of mature and nascent transcripts from human tissues. BMC Biotechnol 13, 99.

Zerbino, D.R., Achuthan, P., Akanni, W., Amode, M.R., Barrell, D., Bhai, J., Billis, K., Cummins, C., Gall, A., Giron, C.G., et al. (2018). Ensembl 2018. Nucleic Acids Res 46, D754-D761.

Zhang, L., Kasif, S., Cantor, C.R., and Broude, N.E. (2004). GC/AT-content spikes as genomic punctuation marks. Proceedings of the National Academy of Sciences $101,16855-16860$.

Zhou, Z., Dang, Y., Zhou, M., Li, L., Yu, C.H., Fu, J., Chen, S., and Liu, Y. (2016). Codon usage is an important determinant of gene expression levels largely through its effects on transcription. Proc Natl Acad Sci U S A 113, E6117-E6125.

Zolotukhin, S., Potter, M., Hauswirth, W.W., Guy, J., and Muzyczka, N. (1996). A "humanized" green fluorescent protein cDNA adapted for high-level expression in mammalian cells. J Virol 70, 4646-4654. 